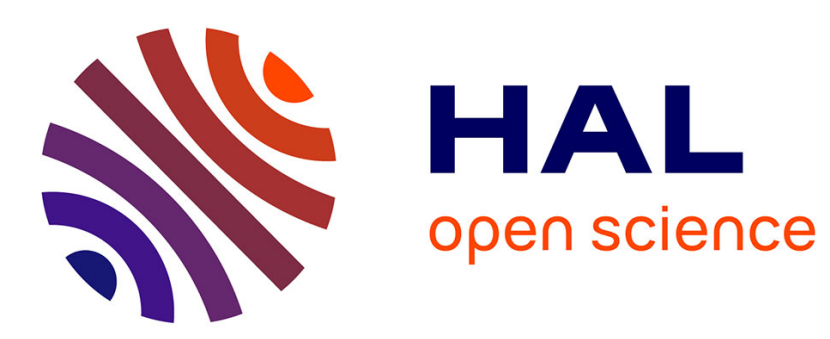

\title{
Wrench-Feasible Workspace of Mobile Cable-Driven Parallel Robots
}

\author{
Tahir Rasheed, Philip Long, Stéphane Caro
}

\section{To cite this version:}

Tahir Rasheed, Philip Long, Stéphane Caro. Wrench-Feasible Workspace of Mobile Cable-Driven Parallel Robots. Journal of Mechanisms and Robotics, 2020, 12 (3), pp.031009. 10.1115/1.4045423 . hal-02379201

\section{HAL Id: hal-02379201 \\ https://hal.science/hal-02379201}

Submitted on 25 Nov 2019

HAL is a multi-disciplinary open access archive for the deposit and dissemination of scientific research documents, whether they are published or not. The documents may come from teaching and research institutions in France or abroad, or from public or private research centers.
L'archive ouverte pluridisciplinaire HAL, est destinée au dépôt et à la diffusion de documents scientifiques de niveau recherche, publiés ou non, émanant des établissements d'enseignement et de recherche français ou étrangers, des laboratoires publics ou privés. 


\title{
Wrench-Feasible Workspace of Mobile Cable-Driven Parallel Robots
}

\author{
Tahir Rasheed \\ École Centrale de Nantes (LS2N), \\ UMR CNRS 6004, 1, rue de la Noë, \\ 44321 Nantes, France \\ Email: tahir.rasheed@Is2n.fr
}

\author{
Philip Long \\ Irish Manufacturing Research, \\ Unit A, Aerodrome Business Park, \\ Rathcoole, Co. Dublin, D24 WCO4, \\ Email: philip.long@imr.ie
}

\author{
Stéphane Caro \\ CNRS, Laboratoire des Sciences du Numérique de Nantes, \\ UMR CNRS n6004, \\ 1 , rue de la Noë, \\ 44321 Nantes Cedex 03, France \\ Email: stephane.caro@ls2n.fr
}

\begin{abstract}
Cable-Driven Parallel Robots hold numerous advantages over conventional parallel robots in terms of high speed and large workspace. Cable Driven Parallel Robots whose workspace can be further increased by the modification of their geometric architecture are known as Reconfigurable Cable Driven Parallel Robots. A novel concept of Reconfigurable Cable Driven Parallel Robots that consists of a classical Cable-Driven Parallel Robot mounted on multiple Mobile Bases is known as Mobile CDPR. This paper proposes a methodology to trace the WrenchFeasible-Workspace of Mobile Cable-Driven Parallel Robots by determining its Available Wrench Set. Contrary to classical Cable-Driven Parallel Robots, we show that the Available Wrench Set of a Mobile Cable-Driven Parallel Robot depends, not only on the cable tension limits, but also on the Static Equilibrium conditions of the Mobile Bases. The Available Wrench Set is constructed by two different approaches known as Convex Hull approach and Hyperplane Shifting Method. Three case studies are carried out for the validation of the proposed methodology. The proposed approach is experimentally validated on a Mobile Cable-Driven Parallel Robot with a point-mass end-effector and two Mobile Bases.
\end{abstract}

\section{Introduction}

Cable-Driven Parallel Robots (CDPRs) are a particular class of conventional parallel manipulators whose rigid limbs are replaced by cables that connect the moving-platform to a fixed base. Each cable is coiled/uncoiled by a motorized winch to displace the moving-platform. The platform motion is generated by appropriately changing all the cable lengths between the moving-platform and the fixed base frame. The lightweight properties of the CDPR give these systems an edge over conventional manipulators and make them suitable for multiple applications such as high acceleration tasks [1], large workspace [2], material handling [3], rehabilitation [4] and logistic applications [5]. Other possible applications are large-scale assembly [6], flight simulators [7] and search-and-rescue operations [8].

In spite of the widespread applications of CDPRs, several challenges remain. For example, collision free trajectory generation must consider all types of collisions, namely, cable/cable, cable/moving-platform, cable/environment and movingplatform/environment collision type [9,10]. Moreover, the location of the CDPR's cable attachment points must be carefully chosen in order to maximize the workspace. Thus, it is important to adapt the robot's geometric architecture according to the task requirements. Such solutions are named as Reconfigurable Cable-Driven Parallel Robots (RCDPRs). The geometric architecture of RCDPRs can be altered by displacing the cable's exit and/or anchor points which can lead to better performances, e.g. lower cable tensions, larger workspace and higher stiffness. The recent work on RCDPRs [11-14] proposed different strategies to determine an optimized robot architecture based on the required task and the robot's environment. Nevertheless, the reconfigurations of existing RCDPRs are usually discrete and performed manually. A concept of CDPRs 


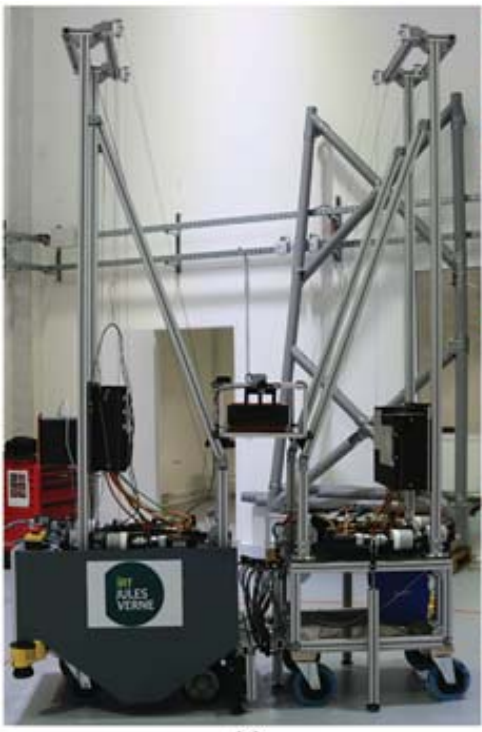

(a)

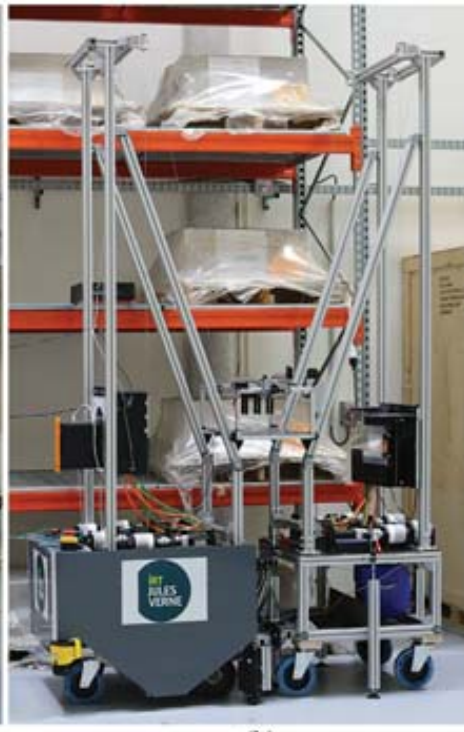

(b)

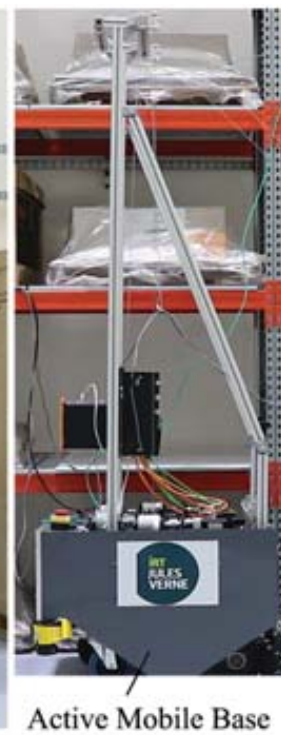

Active Mobile Base

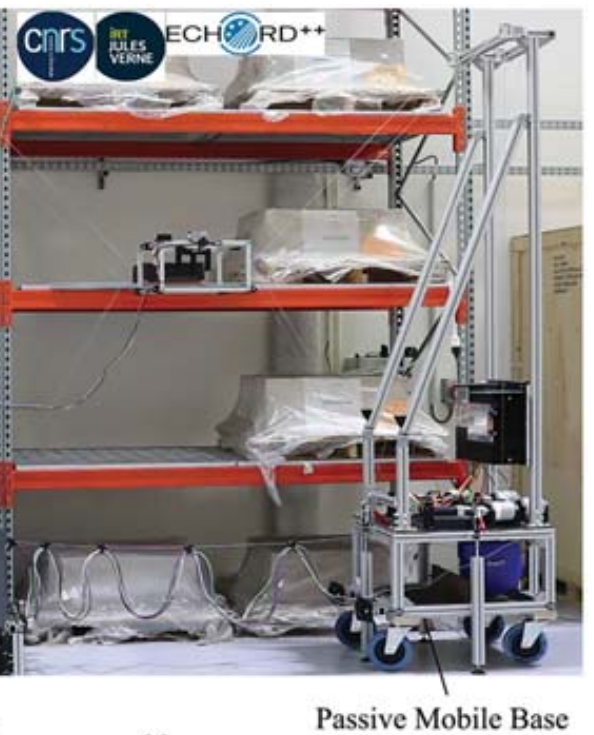

(c)

Fig. 1. FASTKIT prototype (a) Navigation mode (b) Undeployed configuration (c) Deployed configuration at the task location

with base mobility is introduced in [15] for optimizing the orientation workspace and stiffness of the moving-platform. A similar system for optimizing the robot configuration in order to increase the position accuracy of the moving-platform is presented in [16]. A novel concept of Mobile Cable-Driven Parallel Robots (MCDPRs) was introduced in [17]. It uses a combination of mobile bases and a CDPR to create autonomous RCDPRs. A MCDPR is composed of a classical CDPR with $m$ cables and a $n$ degree-of-freedom (DoF) moving-platform mounted on $p$ mobile bases.

The MCDPR prototype "FASTKIT" [18] which was designed and built in the framework of ECHORD++ project $^{1}$ is shown in Fig. 1. FASTKIT is composed of two mobile bases $(p=2)$ with one passive mobile base and one active mobile base. Its moving-platform is pulled by eight cables $(m=8)$ and can perform a six DoF motion type $(n=6)$. The goal of the FASTKIT project is to provide a low cost and versatile robotic solution for logistics using a combination of mobile robots and Cable-Driven Parallel Robot (CDPR). The FASTKIT prototype addresses an industrial need for fast picking and kitting operations in existing storage facilities while being easy to install, maintaining existing infrastructures and covering large areas. The system can navigate autonomously to the area of interest. Once the desired position is attained, the system deploys the CDPR in such a way that its workspace corresponds to the current task specification. The system calculates the required mobile base position from the desired workspace and ensures the controllability of the platform during the deployment. Once the system is successfully deployed, a set of stabilizers is used to ensure the stability of the prototype. Finally, the moving-platform is moved accurately by the CDPR at high velocity over a large area by controlling the cable tensions ${ }^{2}$.

This paper focuses on the Wrench-Feasible Workspace (WFW) of MCDPRs. WFW is defined as the set of platform poses for which the required set of wrenches can be balanced with wrenches generated by the cables, while maintaining the cable tension within the defined limits [19,20]. For a given CDPR configuration, the set of wrenches that can be generated by the cables on the moving-platform is defined as the Available Wrench Set (AWS). Knowing the AWS, we can predict whether the robot has ability to generate the required set of wrenches to perform a task, which can be, for example, the displacement of a sensor mounted on the moving-platform [21] or payload handling [22-24], in a given configuration.

The two methods used to represent the convex polytopes are $\mathscr{V}$-representation, known as the convex hull approach, and $\mathscr{H}$-representation, known as the Hyperplane Shifting Method [25,26]. Contrary to classical CDPRs, the static equilibrium of the mobile bases, characterized by its tipping and sliding conditions, should be considered in addition to the cable tension limits to determine the AWS of MCDPRs. Therefore, the first step is to formulate the static equilibrium conditions of a MCDPR, i.e., the static equilibrium of the moving-platform and the static equilibrium of the mobile bases. These conditions are used to form a cable tension space in order to determine the $\mathscr{V}$-representation of the AWS. The static equilibrium conditions of the mobile bases are mapped into the wrench space by solving the static equilibrium of the moving-platform using Gaussian Elimination Algorithm. The $\mathscr{H}$-representation of the AWS is defined by constructing its facets using cable tension limits and the static equilibrium conditions of the mobile bases expressed in the wrench space. Finally, the $\mathscr{H}$ representation of the AWS is exploited to trace the WFW workspace of MCDPRs. This methodology is illustrated by the flowchart shown in Fig. 2.

As consequence, the paper is organized as follows. Section 2 presents the architecture and parameterization of a

${ }^{1}$ https://www.fastkit-project.eu/

${ }^{2}$ https://www.youtube.com/watch?v=TJSsfiNlvZ4

Corresponding author: Stéphane CARO, email: stephane.caro@1s2n.fr 


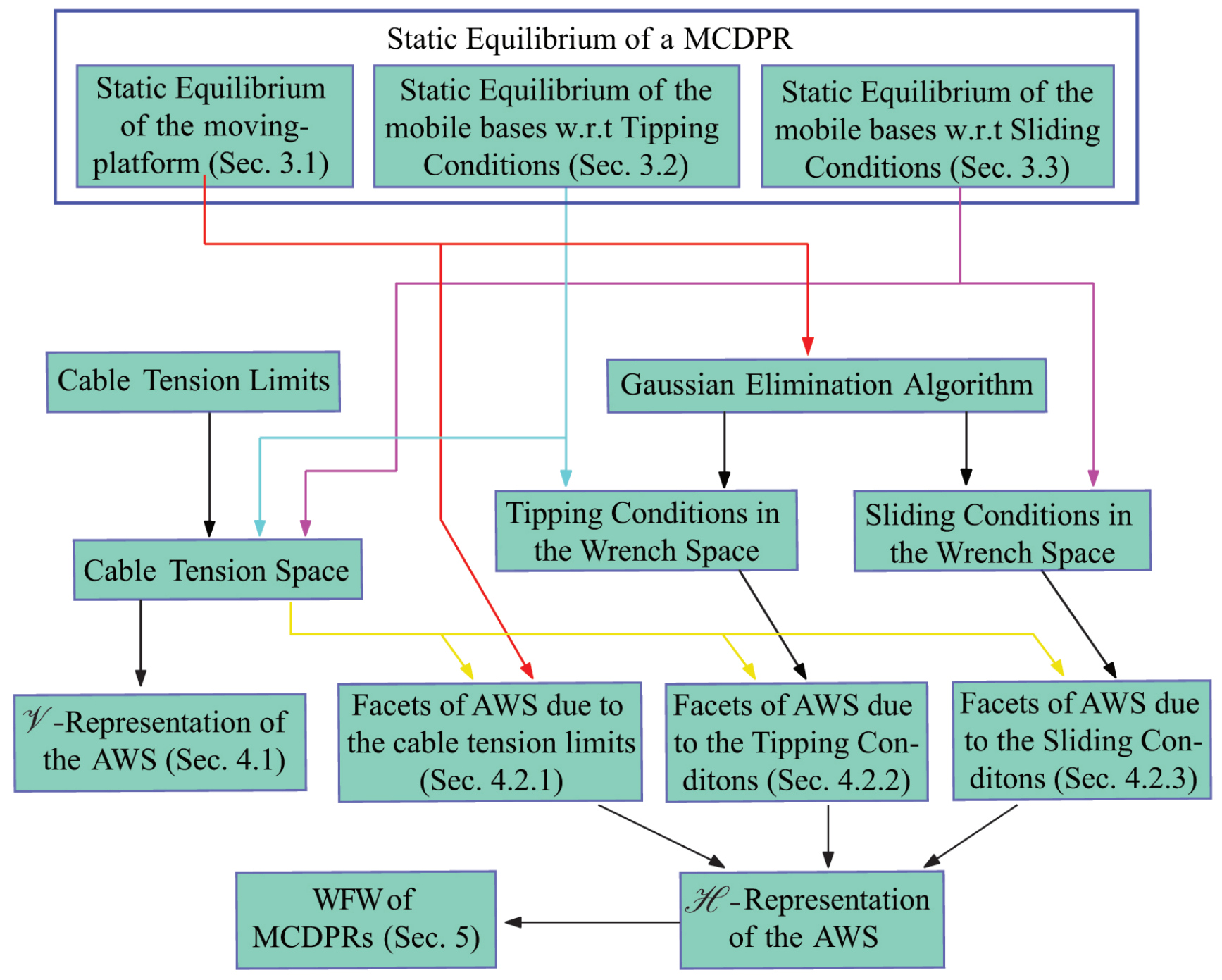

Fig. 2. Methodology used to determine the WFW of a MCDPR

MCDPR. Section 3 expresses the static equilibrium equations of the moving-platform and mobile bases. Those equations are used to determine the manipulator's cable tension space. A methodology to map the static equilibrium conditions of the mobile bases into the wrench space of the moving-platform is described in Sec. 4.2.2. Accordingly, the AWS of MCDPRs is calculated using both the $\mathscr{V}$-representation and the $\mathscr{H}$-representation as explained in Sec. 4. Section 5 explains how the WFW is traced using $\mathscr{H}$-representation of the AWS. Three case studies are given in Sec. 5 to illustrate the theoretical contributions of the paper, along with the experimental validation of the concept. Finally, conclusions are drawn and future work is discussed in Sec. 6.

\section{MCDPR Parameterization}

A MCDPR is composed of a classical CDPR with $m$ cables, a $n$ DoF moving-platform carried by $p$ mobile bases. A MCDPR with $m=8$ cables, $n=6$ DoF moving-platform and $p=4$ mobile bases is shown in Fig. 3 . Let $m_{j}$ be the number cables connected to the $j$ th mobile base denoted as $\mathscr{M}_{j}, j=1, \ldots, p$. The $i$ th cable attached to $\mathscr{M}_{j}$ is named as $\mathscr{C}_{i j}$, $i=1, \ldots, m_{j}$. As a result, $m$ cables are attached to the moving-platform.

$$
m=\sum_{j=1}^{p} m_{j}
$$




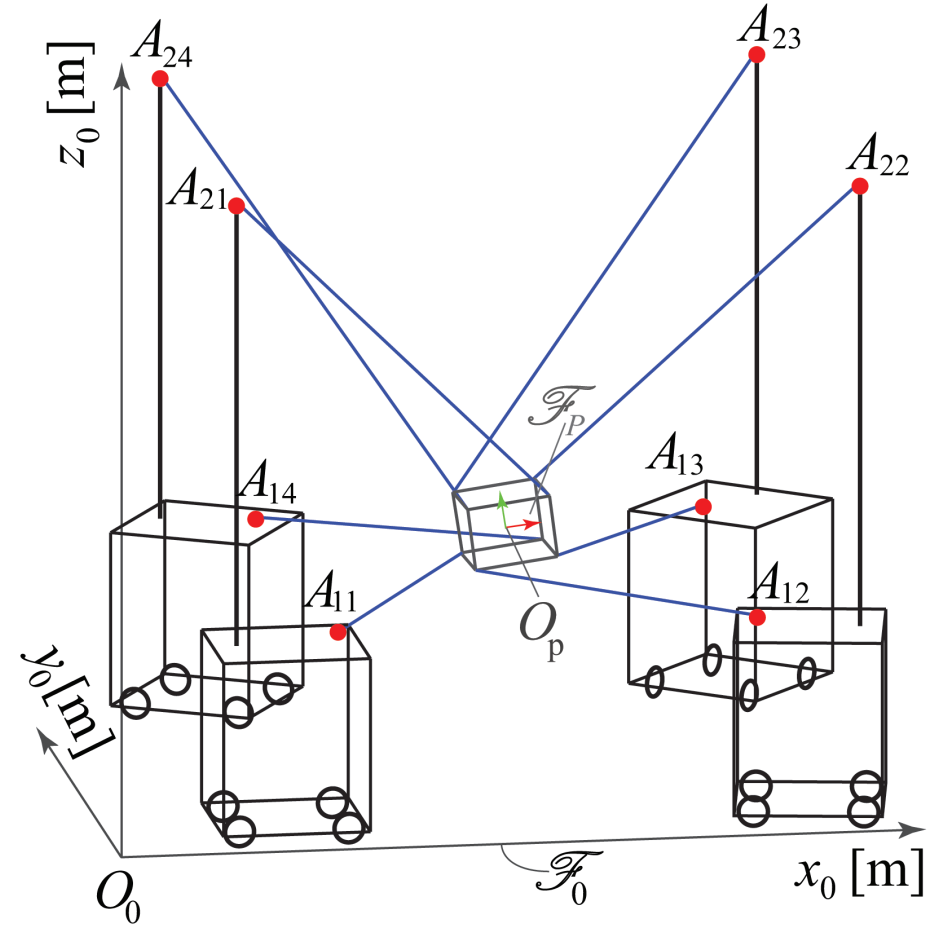

(a)

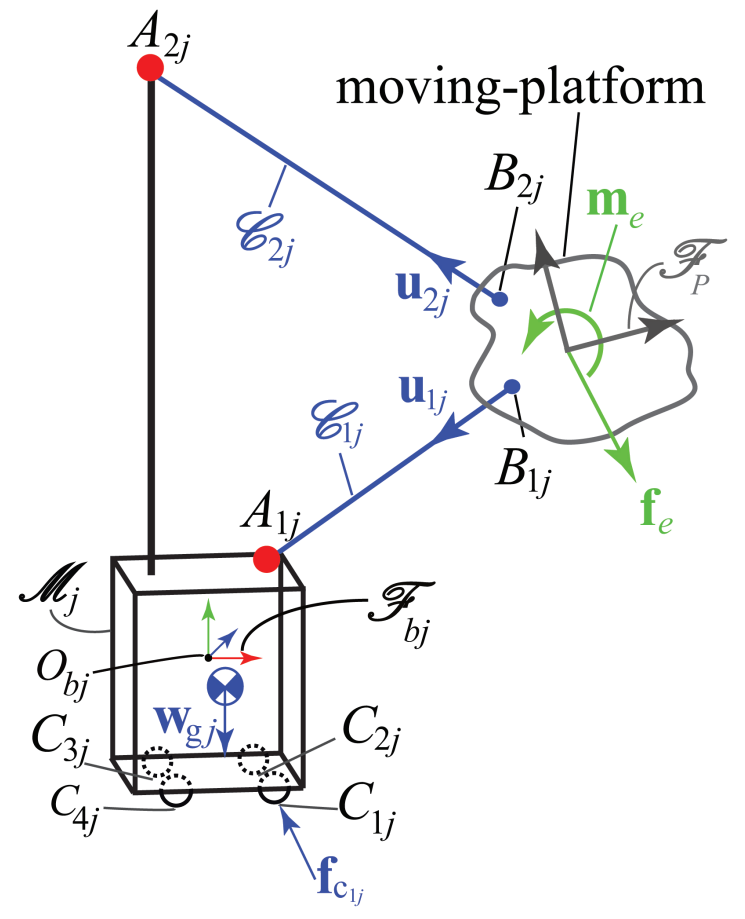

(b)

Fig. 3. (a) A MCDPR with eight cables $(m=8)$ and four mobile bases $(p=4)$. Its moving-platform has six degree-of-freedom ( $\mathrm{n}=6)(\mathrm{b})$ $j$ th mobile base with four wheels $\left(c_{j}=4\right)$.

Let $\mathbf{u}_{i j}$ be the unit vector pointing from the anchor point $B_{i j}$ to the exit point $A_{i j}$ of cable $\mathscr{C}_{i j}$. $\mathbf{t}_{i j}$ is the $\mathscr{C}_{i j}$ cable tension vector expressed as:

$$
\mathbf{t}_{i j}=\mathbf{u}_{i j} t_{i j}
$$

where $t_{i j}$ denotes the tension in the cable $\mathscr{C}_{i j}$. Let $\mathscr{F}_{0}$ be the base frame of origin $O_{0}$ and axes $x_{0}, y_{0}$ and $z_{0}$, respectively. Mobile bases are assumed to be capable of performing two-DoF translational motions along $x_{0}$ and $y_{0}$ and one-DoF rotational motion about an axis parallel to $z_{0}$. The $j$ th mobile base is assumed to have $c_{j}$ wheels. $C_{k j}, k=1, \ldots, c_{j}$ are the contact points between the $j$ th mobile base $\mathscr{M}_{j}$ and the ground. Figure 3(b) illustrates $\mathscr{M}_{j}$ with four wheels $\left(c_{j}=4\right)$.

\section{Static Equilibrium of MCDPRs}

A MCDPR is in a static equilibrium if and only if (iff) its moving-platform and mobile bases are all in static equilibrium. Therefore, the static equilibrium conditions of the moving-platform and the mobile bases of MCDPRs are formulated in this section.

\subsection{Static Equilibrium of the Moving Platform}

The static equilibrium equations of the moving-platform are expressed as [27,28]:

$$
\begin{gathered}
\sum_{j=1}^{p} \sum_{i=1}^{m_{j}} \mathbf{u}_{i j} t_{i j}=\mathbf{f}, \\
\sum_{j=1}^{p} \sum_{i=1}^{m_{j}} \mathbf{c}_{r i j} t_{i j}=\mathbf{m},
\end{gathered}
$$


where $\mathbf{f}=\left[f^{x}, f^{y}, f^{z}\right]^{T}$ and $\mathbf{m}=\left[m^{x}, m^{y}, m^{z}\right]^{T}$ denote the forces and moments applied by the cables onto the moving platform. $\mathbf{c}_{r i j}$ gives the direction of the actuation moment applied by the cable $\mathscr{C}_{i j}$ onto the moving-platform expressed as:

$$
\mathbf{c}_{r i j}=\mathbf{r}_{i j} \times \mathbf{u}_{i j}
$$

where $\mathbf{r}_{i j}$ is a vector pointing from the origin $O_{P}$ of the moving-platform frame $\mathscr{F}_{P}$ to the cable anchor point $B_{i j}$. The static equilibrium of the moving-platform is expressed in a matrix form as:

$$
\mathbf{W t}=\mathbf{w}
$$

where $\mathbf{W}$ is a $(n \times m)$ wrench matrix mapping the cable tension vector $\mathbf{t} \in \mathbb{R}^{m}$ onto the wrenches $\mathbf{w} \in \mathbb{R}^{n}$ applied by the cables onto the moving-platform.

$$
\mathbf{W}=\left[\begin{array}{lllll}
\mathbf{W}_{1} & \ldots & \mathbf{W}_{j} & \ldots & \mathbf{W}_{p}
\end{array}\right], \quad \mathbf{w}=\left[\begin{array}{c}
\mathbf{f} \\
\mathbf{m}
\end{array}\right], \quad \mathbf{t}=\left[\begin{array}{c}
\mathbf{t}_{1} \\
\vdots \\
\mathbf{t}_{j} \\
\vdots \\
\mathbf{t}_{p}
\end{array}\right]
$$

$\mathbf{W}_{j}$ is a $\left(n \times m_{j}\right)$-dimensional matrix whose columns are the actuation wrenches exerted by the cables attached to $\mathscr{M}_{j}$. $\mathbf{t}_{j}$ is a $m_{j}$-dimensional cable tension vector corresponding to the cables connected to $\mathscr{M}_{j}$.

$$
\begin{aligned}
& \mathbf{t}_{j}=\left[\begin{array}{lllll}
t_{1 j} & \ldots & t_{i j} & \ldots & t_{m_{j} j}
\end{array}\right]^{T}, \\
& \mathbf{W}_{j}=\left[\begin{array}{lllll}
\mathbf{w}_{1 j} & \ldots & \mathbf{w}_{i j} & \ldots & \mathbf{w}_{m_{j} j}
\end{array}\right] .
\end{aligned}
$$

$\mathbf{w}_{i j}$ is the actuation wrench generated by the cable $\mathscr{C}_{i j}$ and is expressed as:

$$
\mathbf{w}_{i j}=\left[\begin{array}{c}
\mathbf{u}_{i j} \\
\mathbf{c}_{r i j}
\end{array}\right] .
$$

The cable tension are all bounded between a minimum and positive tension $\underline{t}_{i j}$ and a maximum tension $\bar{t}_{i j}$

$$
\underline{t}_{i j} \leq t_{i j} \leq \bar{t}_{i j}, \quad i=1, \ldots, m_{j}, j=1, \ldots, p
$$

\subsection{Static Equilibrium of Mobile Bases}

The static equilibrium of a wheeled mobile base can be characterized by its tipping and sliding conditions. To obtain the effect of these conditions on the wrench abilities of the moving platform, the latter must be first expressed in terms of the cable tensions. From Fig. 3(b), the equilibrium conditions of $\mathscr{M}_{j}$ are expressed as [17]:

$$
\begin{gathered}
\mathbf{w}_{g j}+\sum_{k=1}^{c_{j}} \mathbf{f}_{c_{k j}}-\sum_{i=1}^{m_{j}} \mathbf{t}_{i j}=\mathbf{0} \\
\mathbf{g}_{j} \times \mathbf{w}_{g j}+\sum_{k=1}^{c_{j}} \mathbf{c}_{k j} \times \mathbf{f}_{c_{k j}}-\sum_{i=1}^{m_{j}} \mathbf{b}_{i j} \times \mathbf{t}_{i j}=\mathbf{0}
\end{gathered}
$$


where $\mathbf{f}_{c_{k j}}=\left[f_{c_{k j}}^{x}, f_{c_{k j}}^{y}, f_{c_{k j}}^{z}\right]$ denotes the ground contact force at $C_{k j} . \mathbf{g}_{j}$ denotes the Cartesian coordinate vector of the center of gravity $G_{j}$. $\mathbf{c}_{k j}$ denotes the Cartesian coordinate vector of the wheel contact point $C_{k j}$. $\mathbf{b}_{i j}$ denotes the Cartesian coordinate vector of the platform attachment points $B_{i j} . \mathbf{w}_{g j}$ is the weight vector of $\mathscr{M}_{j}$. The aforementioned vectors are all expressed in the base frame $\mathscr{F}_{0}$.

Equations (10a) and (10b) represent the classical static equilibrium conditions of $\mathscr{M}_{j}$ in terms of the cable tensions. From those equations, Secs. 3.2.1 and 3.2.2 formulate the static equilibrium conditions corresponding to the tipping and sliding conditions of mobile bases in terms of cable tensions.

\subsubsection{Tipping conditions of the Mobile Bases}

The equilibrium towards the tipping of a wheeled robot is defined by an index named Zero-Moment Point (ZMP) [29-31]. It is the point where the moment of ground contact forces is reduced to the pivoting moment of friction forces about an axis normal to the ground. It amounts to the point where the sum of the moments due to planar ground reaction forces is null. Let $C_{j}$ denotes the ZMP of $\mathscr{M}_{j}$. ZMP can be calculated from the wheel contact points $C_{k j}, k=1, \ldots, c_{j}$ as [32]

$$
\mathbf{c}_{j}=\frac{\sum_{k=1}^{c_{j}} \mathbf{c}_{k j} f_{c_{k j}}^{z}}{\sum_{k=1}^{c_{j}} f_{c_{k j}}^{z}} .
$$

where $\mathbf{c}_{j}$ represents the Cartesian coordinates of the ZMP of $j$ th mobile base. The tipping conditions depend on the moments generated at the boundaries of the mobile base footprint. The footprint is formed by joining the contact points $C_{k j}, k=$ $\left\{1, \ldots, c_{j}\right\}$ selected counter-clockwise (See Fig. 4(a)). The boundary between the two consecutive contact points $C_{k j}$ and $C_{k+1 j}$ of $\mathscr{M}_{j}$ is denoted as $\mathscr{L}_{C_{k j}}$ of unit vector $\mathbf{u}_{C_{k j}}$. Let $m_{C_{k j}}$ be the moment generated about $\mathscr{L}_{C_{k j}}$ at the instant when $\mathscr{M}_{j}$ loses contact with the ground at the points which do not form the boundary $\mathscr{L}_{C_{k j}}$,

$$
m_{C_{k j}}=\mathbf{u}_{C_{k j}}^{T}\left(\left(\mathbf{g}_{j}-\mathbf{c}_{k j}\right) \times \mathbf{w}_{g j}\right)+\sum_{i=1}^{m_{j}} \mathbf{u}_{C_{k j}}^{T}\left(\left(\mathbf{c}_{k j}-\mathbf{b}_{i j}\right) \times \mathbf{u}_{i j}\right) t_{i j}, \quad k=1, \ldots, c_{j}
$$

For $\mathscr{M}_{j}$ to be in static equilibrium, $m_{C_{k j}}, k=1, \ldots, c_{j}$, should be negative, namely,

$$
m_{C_{k j}} \leq 0, \quad k=1, \ldots, c_{j}
$$

Equation (13) defines the tipping conditions of $\mathscr{M}_{j}$ expressed in terms of the cable tensions $t_{i j}, i=1, \ldots, m_{j}$. Each $k$ th boundary $\mathscr{L}_{C_{k j}}$ is associated with a single tipping condition. It means that the total number of tipping conditions to be satisfied for $\mathscr{M}_{j}$ to be in static equilibrium is equal to the number of wheels, $c_{j}$.

\subsubsection{Sliding conditions of the Mobile Bases}

Sliding conditions are defined by a friction cone at each wheel of the mobile base. For the mobile base to be in static equilibrium, the ground contact force $\mathbf{f}_{c_{k j}}$ at $C_{k j}$ must be within the corresponding $k$ th friction cone. The frictional effects due to the wheel contact points can be represented as a single friction cone located at the ZMP expressed as,

$$
\sqrt{{ }^{b j} f_{c_{j}}^{x^{2}}+{ }^{b j} f_{c_{j}}^{y}{ }^{2}} \leq \mu^{b j} f_{c_{j}}^{z}
$$

where ${ }^{b j} \mathbf{f}_{c_{j}}=\left[{ }^{b j} f_{c_{j}}^{x}{ }^{b j} f_{c_{j}}^{y}{ }^{b j} f_{c_{j}}^{z}\right]^{T}$ represents the ground contact force at ZMP expressed in frame $\mathscr{F}_{b j}$. In this paper, the sliding condition is linearized and the friction cone becomes a four-sided friction pyramid [33,34] as shown in Fig. 4(b). Consequently, Eq. (14) is simplified as follows:

$$
\left[\begin{array}{ccc}
1 & 0 & -\mu \\
-1 & 0 & -\mu \\
0 & 1 & -\mu \\
0 & -1 & -\mu
\end{array}\right]{ }^{b j} \mathbf{f}_{c_{j}} \leq \mathbf{0}_{4}
$$

where $\mu$ denotes the friction coefficient between the ground and the wheels of $\mathscr{M}_{j}$. 


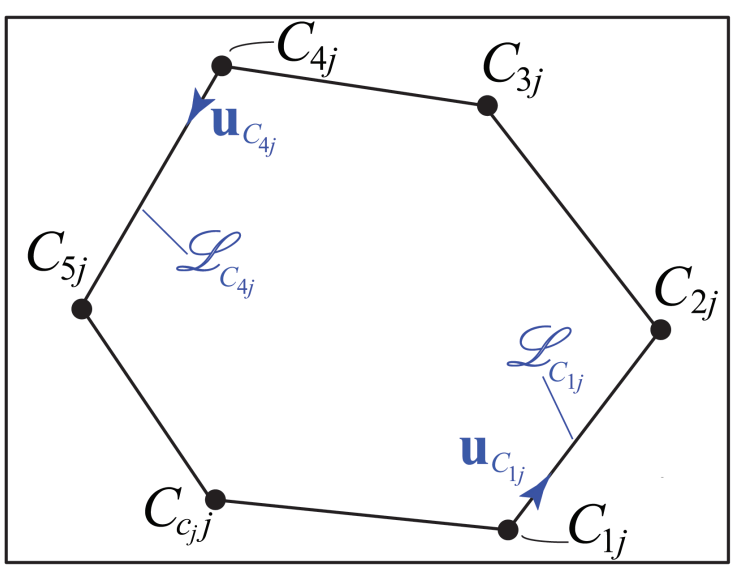

(a)

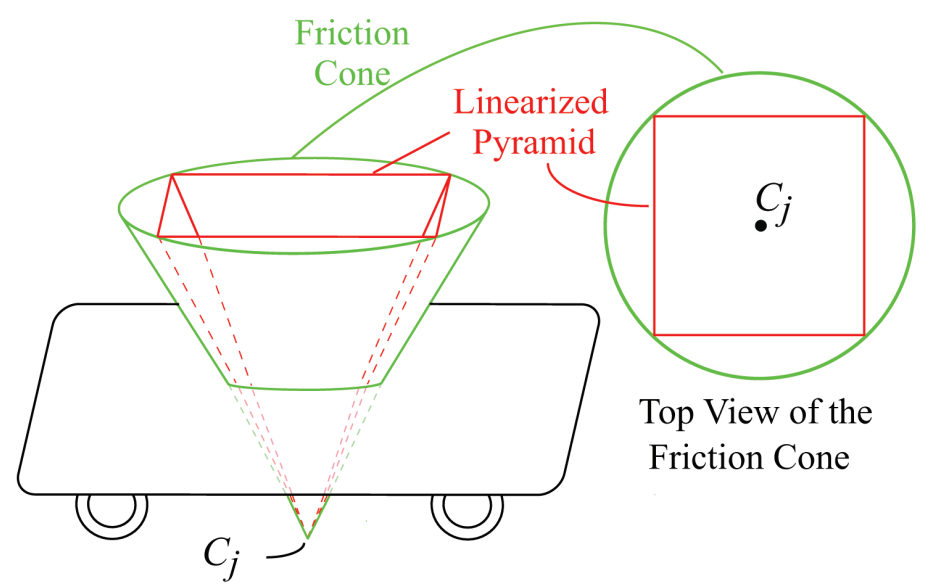

(b)

Fig. 4. (a) Footprint of $\mathscr{M}_{j}$ with $c_{j}=6$ wheels (b) Linearized friction pyramid at ZMP $\left(C_{j}\right)$.

Equation (15) can be represented in the base frame $\mathscr{F}_{0}$ as

$$
\mathbf{E}_{f}{ }^{0} \mathbf{f}_{c_{j}} \leq \mathbf{0}_{4}
$$

where

$$
{ }^{0} \mathbf{f}_{c_{j}}={ }^{0} \mathbf{R}_{b j}{ }^{b j} \mathbf{f}_{c_{j}}, \quad \mathbf{E}_{f}=\left[\begin{array}{ccc}
1 & 0 & -\mu \\
-1 & 0 & -\mu \\
0 & 1 & -\mu \\
0 & -1 & -\mu
\end{array}\right]{ }^{b j} \mathbf{R}_{0} .
$$

${ }^{0} \mathbf{R}_{b j}$ denotes the rotation matrix from $\mathscr{F}_{0}$ to $\mathscr{F}_{b j}$. Accordingly, ${ }^{b j} \mathbf{R}_{0}$ is the inverse of ${ }^{0} \mathbf{R}_{b j}$.

${ }^{0} \mathbf{f}_{c_{j}}=\left[\begin{array}{llll}{ }^{0} f_{c_{j}}^{x} & 0 & f_{c_{j}}^{y} & { }^{0} f_{c_{j}}^{z}\end{array}\right]^{T}$ denotes the ground contact forces at ZMP expressed in $\mathscr{F}_{0}$ :

$$
{ }^{0} \mathbf{f}_{c_{j}}=\sum_{k=1}^{c_{j}} \mathbf{f}_{c_{k j}}=-\mathbf{w}_{g j}+\sum_{i=1}^{m_{j}} \mathbf{u}_{i j} t_{i j}
$$

Substituting Eq. (18) in Eq. (16) yields:

$$
\sum_{i=1}^{m_{j}} \mathbf{E}_{f} \mathbf{u}_{i j} t_{i j}-\mathbf{E}_{f} \mathbf{w}_{g j} \leq \mathbf{0}_{4}
$$

As a result, Eq. (19) defines the sliding conditions associated with $\mathscr{M}_{j}$ formulated in the form of a linearized friction pyramid in terms of the cable tensions $t_{i j}, i=1, \ldots, m_{j}$.

\section{Available Wrench Set for MCDPRs}

For a given pose, the set of wrenches a mechanism can generate is defined as Available Wrench Set (AWS) [35]. For classical CDPRs, AWS $\mathscr{A}_{C D P R}$ depends uniquely on the robot's geometric architecture and the cable tension limits [?,25], expressed as:

$$
\mathscr{A}_{C D P R}=\left\{\left[\begin{array}{c}
\mathbf{f} \\
\mathbf{m}
\end{array}\right] \in \mathbb{R}^{n} \mid\left[\begin{array}{c}
\mathbf{f} \\
\mathbf{m}
\end{array}\right]=\mathbf{W} \boldsymbol{t}, \underline{t}_{i j} \leq t_{i j} \leq \bar{t}_{i j}, i=1, \ldots, m_{j}, j=1, \ldots, p\right\} .
$$




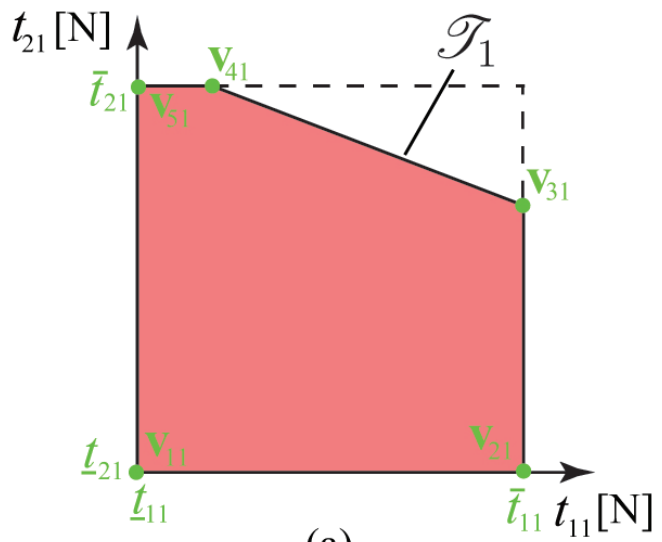

(a)

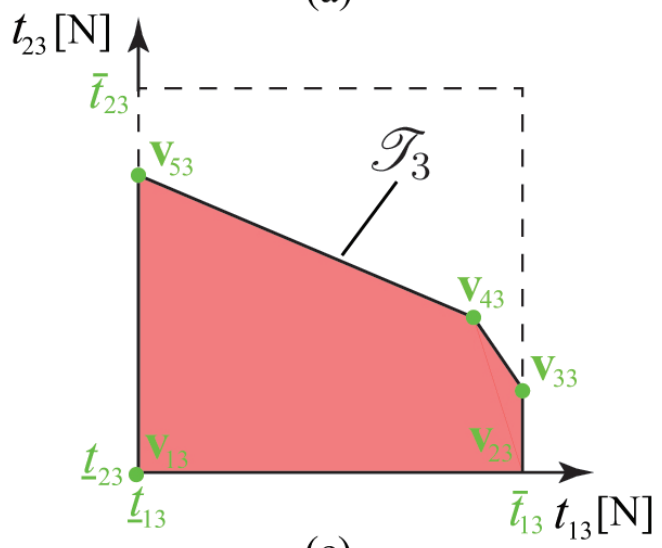

(c)

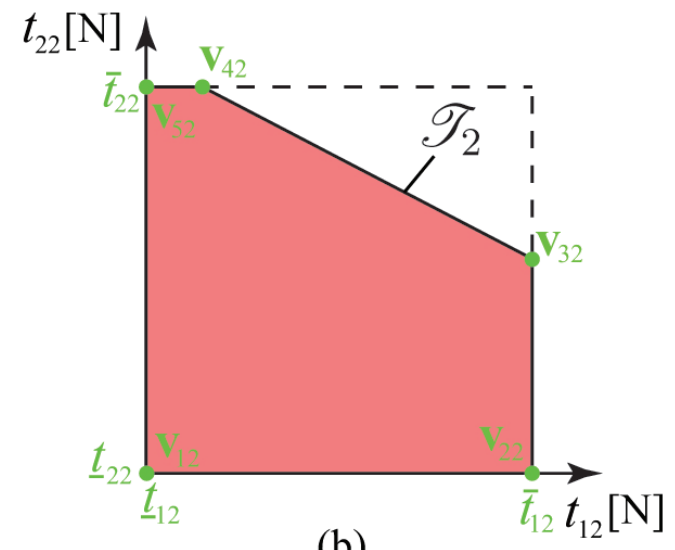

(b)

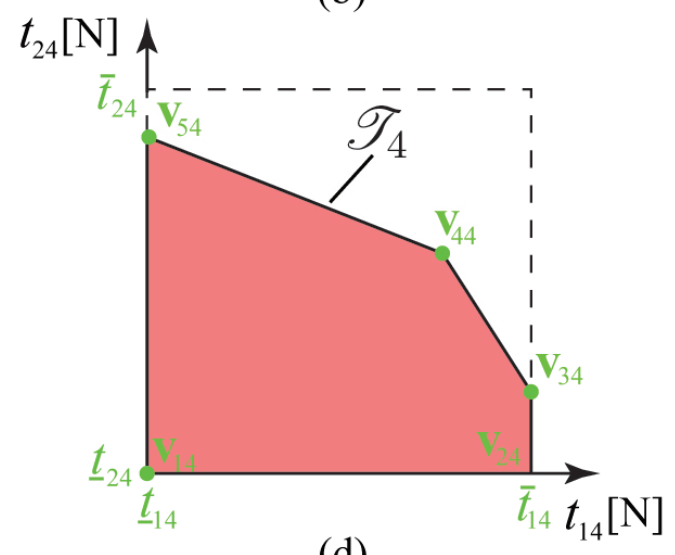

(d)

Fig. 5. Tension space associated to (a) $\mathscr{M}_{1}$, (b) $\mathscr{M}_{2}$,(c) $\mathscr{M}_{3}$,(d) $\mathscr{M}_{4}$ considering both the cable tension limits and the static equilibrium of the mobile bases

On the contrary, the AWS of MCDPRs cannot be fully characterized by the cable tension limits as the mobile bases static equilibrium conditions must be considered. Hence, the AWS $\mathscr{A}$ of a MCDPR is defined as:

$$
\begin{aligned}
\mathscr{A}= & \left\{\left[\begin{array}{c}
\mathbf{f} \\
\mathbf{m}
\end{array}\right] \in \mathbb{R}^{n} \mid\left[\begin{array}{c}
\mathbf{f} \\
\mathbf{m}
\end{array}\right]=\mathbf{W} \boldsymbol{t}, \underline{t}_{i j} \leq t_{i j} \leq \bar{t}_{i j}, m_{C_{k j}} \leq 0,\right. \\
& \left.\mathbf{E}_{f}{ }^{0} \mathbf{f}_{c_{j}} \leq \mathbf{0}_{4}, i=1, \ldots, m_{j}, k=1, \ldots, c_{j}, j=1, \ldots, p\right\} .
\end{aligned}
$$

By adding the additional conditions associated with the static equilibrium of the mobile bases, $\mathscr{A}$ is no longer a zonotope, but a convex polytope [35]. The two widely used approaches to characterize such convex polytopes are $\mathscr{V}$-representation, known as the Convex Hull approach, and $\mathscr{H}$-representation, known as the Hyperplane Shifting Method (HSM) [26]. $\mathscr{V}$ representation is preferred for visualizing the AWS but is computationally more expensive than $\mathscr{H}$-representation, which is used to determine the relation between the AWS and the required wrenches to perform a task. The convex-hull approach uses the vertices of the cable tension space to determine the vertices of AWS and forms the boundary of the convex polytope described in Sec. 4.1. HSM allows us to determine the AWS geometrically by characterizing the facets of the polytope as explained in Sec. 4.2. This may be exploited to trace the WFW of MCDPRs.

\subsection{Convex Hull Method}

For MCDPRs, AWS takes the form of a convex polytope and is the image of the tension space under the linear mapping of the wrench matrix $\mathbf{W}[?, 25,35]$. Thus $\mathscr{V}$-representation defines the AWS of MCDPRs by finding the set of vertices forming the boundary of the convex polytope. The vertices of the AWS are obtained by mapping the tension space vertices into the wrench space under $\mathbf{W}$. Thus the tension space vertices of a MCDPR should first be defined.

The cable tension space defines the region of acceptable cable tensions which maintains the static equilibrium of a MCDPR. The tension space of classical CDPRs is only formed by the cable tension limits and takes the form of a $m$ - 
dimensional hypercube [25]. For MCDPRs, the $j$ th mobile base has its own independent $m_{j}$-dimensional tension space associated only with its attached cables of tensions $t_{i j}, i=1, \ldots, m_{j}$. Therefore, the cable tension space of $\mathscr{M}_{j}$, denoted as $\mathscr{T}_{j}$, is formed by mapping the static equilibrium conditions of the latter defined by Eqs. (13) and (19) on the $m_{j}$-dimensional tension space formed by the cable tension limits $\underline{t}_{i j}$ and $\bar{t}_{i j}$. Figure 5 illustrates the tension space associated with each mobile base of the MCDPR under study as shown in Fig 3(a).

Without loss of generality, let's assume that $\mathscr{T}_{j}$ is composed of $v_{j}$ vertices. Let the $m_{j}$-dimensional coordinate vector of the $d$ th vertex of $\mathscr{T}_{j}, d=1, \ldots, v_{j}$ be denoted as $\mathbf{v}_{d j}$. The vertices of $\mathscr{T}_{j}$ and their coordinates can be obtained using the Double Description Method [36], which exploits the static equilibrium conditions of $\mathscr{M}_{j}$ and the bounds on the cable tensions $t_{i j}, i=1, \ldots, m_{j}$. Let $\mathscr{V}_{j}$ be the set of vertices of $\mathscr{T}_{j}$,

$$
\mathscr{V}_{j}=\left\{\mathbf{v}_{d j}\right\}, \quad d=1, \ldots, v_{j}
$$

The coordinates of the vertices for $\mathscr{T}_{j}$ can be expressed in a matrix form as:

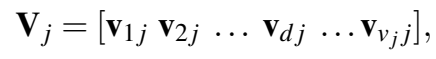

where $\mathbf{V}_{j}$ is a $\left(m_{j} \times v_{j}\right)$ matrix containing the coordinates of the vertices of $\mathscr{T}_{j}$. Let $v$ be the total number of vertices formed by the $m$ cables, which is defined by the product of the number of vertices of the tension space associated to each mobile base, namely,

$$
v=\prod_{j=1}^{p} v_{j} .
$$

The vertices of the tension space of a MCDPR can be obtained by taking the Cartesian product between the vertices of $\mathscr{V}_{j}$, $j=1, \ldots, p$. Let $\mathscr{V}$ denote the set of all vertices of the tension space for a given MCDPR. Let $\mathbf{V}$ be a $(m \times v)$-matrix denoting the coordinates of the vertices in $\mathscr{V}$ expressed as:

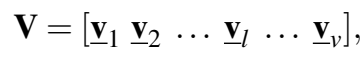

where $l=1, \ldots, v \cdot \underline{\mathbf{v}}_{l}$ is a $m$-dimensional vector representing the coordinates of the $l$ th vertex of the MCDPR cable tension space noted as $\mathscr{T}$. The image of AWS is constructed from $\mathbf{V}$ under the linear mapping of the wrench matrix $\mathbf{W}$ expressed as:

$$
\mathbf{W}_{A}=\mathbf{W V}
$$

where $\mathbf{W}_{A}$ is a $(n \times v)$-matrix representing the image of the vertices of the MCDPR tension space into the wrench space. The convex hull of the points whose coordinate vectors are the columns of $\mathbf{W}_{A}$ leads to the AWS of the MCDPR using a numerical procedure known as quickhull [37].

\subsection{Hyperplane Shifting Method}

The Hyperplane Shifting Method (HSM) is a geometric approach, which defines a convex polytope as the intersection of the half-spaces bounded by its hyperplanes [?]. The classical HSM used to characterize the AWS of the CDPRs is explained in [?,25]. However, due to the additional static equilibrium conditions, the classical HSM is not sufficient to fully characterize the AWS of MCDPRs [35]. As a consequence, this section introduces an improved version of HSM while taking into account the static equilibrium conditions of the MCDPR mobile bases in addition to the cable tension limits.

The determination of the hyperplanes of the AWS when the tension space is not a hypercube but is a convex polytope was addressed in [35]. However, it only deals with planar MCDPRs having a point-mass end-effector and only considers the tipping conditions of the mobile bases. On the contrary, this paper takes into account both the tipping and sliding conditions of spatial MCDPRs. Section 4.2.1 characterizes the facets of the AWS denoted as $\mathscr{H}_{q}^{+}, \mathscr{H}_{q}^{-}$, $q=1, \ldots, C_{n-1}^{m}=\frac{m !}{(m-n+1) !(n-1) !}$, associated with the cable tension limits of MCDPRs. To express the facets of the AWS associated with the static equilibrium conditions of $\mathscr{M}_{j}$, it is required to map the latter into the wrench space. Therefore, Sec. 4.2.2 presents the adopted methodology to map the static equilibrium conditions of $\mathscr{M}_{j}$ into the wrench space. Eventually, Secs. 4.2.3 and 4.2.4 present the hyperplanes associated with both the tipping and sliding conditions of $\mathscr{M}_{j}$. 


\subsubsection{Hyperplanes associated with Cable Tension Limits}

For classical CDPRs, AWS takes the form of a zonotope by considering only the constraints associated with the cable tension limits [?,25]. The facets of the zonotope are formed by the set of vectors $\beta_{i j} \mathbf{u}_{i j} \Delta t_{i j}, 0 \leq \beta_{i j} \leq 1 . \Delta t_{i j}$ represents the difference between the maximum and minimum cable tension limits of $\mathscr{C}_{i j}$, expressed as:

$$
\Delta t_{i j}=\bar{t}_{i j}-\underline{t}_{i j}
$$

The shape of the zonotope is formed by the directions of the cable unit vectors $\mathbf{u}_{i j}$ while the size of the zonotope depends on $\Delta t_{i j}$. However, $\Delta t_{i j}$ is no longer a constant for MCDPRs as illustrated in Fig. 5. The property of a zonotope having parallel facets still holds as the shape of the facets are defined by the cable unit vectors $\mathbf{u}_{i j}$. However the location of the hyperplanes forming the facets of the AWS is modified leading to a convex polytope with parallel facets. Thus, the hyperplanes associated with the cable tension limits for MCDPRs are determined using classical HSM detailed in [?,25] and by considering the modified tension space.

The pairs of parallel hyperplanes are determined by the sets of $(n-1)$ column vectors of $\mathbf{W}[?, 25]$. As a consequence, the cable tension limits leads to $C_{n-1}^{m}$ hyperplanes $\left(\mathscr{H}_{q}^{+}, \mathscr{H}_{q}^{-}, q=1, \ldots, C_{n-1}^{m}\right)$. The first step is to obtain the orientation of those hyperplanes by taking $n-1$ linear combinations out of $m$ columns of $\mathbf{W}$. The $q$ th combination, ${ }^{c} \mathbf{W}^{q}$ is a $(n \times n-1)$-matrix containing $(n-1)$ columns of $\mathbf{W}$. The remaining $m-n+1$ columns of $\mathbf{W}$ are denoted as ${ }^{d} \mathbf{W}^{q}$ such that,

$$
\mathbf{W}=\left[{ }^{c} \mathbf{W}^{q d} \mathbf{W}^{q}\right]
$$

The orientation of $\mathscr{H}_{q}^{+}, \mathscr{H}_{q}^{-}$is defined by the $n$-dimensional unit vector $\mathbf{e}_{q}$ orthogonal to its facets, expressed as

$$
\mathbf{e}_{q}=\frac{\mathbf{r}_{q}}{\left\|\mathbf{r}_{q}\right\|}
$$

where $\mathbf{r}_{q}$ is a $n$-dimension vector expressed as the linear combination of the columns of ${ }^{c} \mathbf{W}^{q}$. The position of $\mathscr{H}_{q}^{+}, \mathscr{H}_{q}^{-}$ is given by the projection of the MCDPR tension space vertices on $\mathbf{e}_{q}$. Let $\mathbf{l}_{q}$ be a $m$-dimensional vector representing the projection of $\mathbf{W}$ on $\mathbf{e}_{q}$,

$$
\mathbf{l}_{q}=\mathbf{W}^{T} \mathbf{e}_{q}
$$

It is noteworthy that the projection of the actuation wrenches in ${ }^{c} \mathbf{W}$ is null as they are orthogonal to $\mathbf{e}_{q}$. Let $h_{q}^{+}, h_{q}^{-}$be the maximum and minimum combinations of $\mathbf{l}_{q}$ with the coordinates of the MCDPR tension space vertices, namely,

$$
h_{q}^{+}=\max \left(\underline{\mathbf{v}}_{l}^{T} \mathbf{l}_{q}, \quad l=1, \ldots, v\right) ; \quad h_{q}^{-}=\min \left(\underline{\mathbf{v}}_{l}^{T} \mathbf{l}_{q}, \quad l=1, \ldots, v\right) .
$$

To completely characterize the location of the hyperplanes, a point $p_{q}^{+}\left(p_{q}^{-}\right.$, resp.) must be defined on $\mathscr{H}_{q}^{+}\left(\mathscr{H}_{q}^{-}\right.$, resp.), expressed as:

$$
\mathbf{p}_{q}^{+}=h_{q}^{+} \mathbf{e}_{q}+\mathbf{W} \underline{\mathbf{t}} ; \quad \mathbf{p}_{q}^{-}=h_{q}^{-} \mathbf{e}_{q}+\mathbf{W} \underline{\mathbf{t}},
$$

where $\underline{\mathbf{t}}=\left[\underline{t}_{11}, \ldots, \underline{t}_{i j}, \ldots, \underline{t}_{m_{p}}\right]^{T}$ is a $m$-dimensional vector containing the cable tension lower bounds. Wt defines the wrench generated by the smallest cable tensions. The position of $\mathscr{H}_{q}^{+}\left(\mathscr{H}_{q}^{-}\right.$, resp. $)$is determined by a shifting distance $d_{q}^{+}\left(d_{q}^{-}\right.$, resp.) along $\mathbf{e}_{q}$ from the origin, expressed as:

$$
d_{q}^{+}=\mathbf{e}_{q}^{T} \mathbf{p}_{q}^{+} ; \quad d_{q}^{-}=\mathbf{e}_{q}^{T} \mathbf{p}_{q}^{-}
$$

Finally, the respective pairs of hyperplanes $\mathscr{H}_{q}^{+}, \mathscr{H}_{q}^{-}$are expressed as:

$$
\mathscr{H}_{q}^{+}: \mathbf{e}_{q}^{T}\left[\begin{array}{c}
\mathbf{f} \\
\mathbf{m}
\end{array}\right] \leq d_{q}^{+} ; \quad \mathscr{H}_{q}^{-}:-\mathbf{e}_{q}^{T}\left[\begin{array}{c}
\mathbf{f} \\
\mathbf{m}
\end{array}\right] \leq d_{q}^{-}
$$

The above procedure is repeated to determine the $C_{n-1}^{m}$ pairs of hyperplanes associated to the $m$ cables of a MCDPR. 


\subsubsection{Static Equilibrium of $\mathscr{M}_{j}$ in Wrench Space}

To map the static equilibrium conditions associated with $\mathscr{M}_{j}$ into the wrench space, the system of linear equations defined in Eq. (5) should be solved. In case $m=n$ and $\mathbf{W}$ is full rank, the cable tensions $t_{i j}, i=1, \ldots, m_{j}$ are expressed as,

$$
\mathbf{t}=\mathbf{W}^{-1} \mathbf{w}
$$

The solutions of the cable tensions $t_{i j}, i=1, \ldots, m_{j}$ extracted from Eq. (35) are substituted in static equilibrium the conditions of $\mathscr{M}_{j}$ defined in Eqs. (13) and (19) to map the latter into the wrench space. Thus, if $m=n$, each static equilibrium condition in the tension space will also generate a single condition into the wrench space.

On the contrary, if $m>n$, there will exist $C_{n}^{m}=\frac{m !}{(m-n) ! n !}$ number of possible solutions for $t_{i j}$ to map the static equilibrium conditions associated with $\mathscr{M}_{j}$ into the wrench space. As a consequence, there exists $C_{n}^{m}$ number of $(n \times n)$ square submatrices of the wrench matrix $\mathbf{W}$ denoted as ${ }^{a} \mathbf{W}^{s}, s=1, \ldots, C_{n}^{m}$ containing $n$ columns of $\mathbf{W}$. Let ${ }^{a} \mathbf{t}^{s}$ be a $n$-dimensional vector containing the cable tensions associated with the actuation wrenches of ${ }^{a} \mathbf{W}^{s}$. Let ${ }^{a} \mathbf{V}^{s}$ be a $(n \times v)$ matrix denoting the coordinates of the tension space vertices of ${ }^{a} \mathbf{t}^{s} .{ }^{a} \mathbf{V}^{s}$ is defined by extracting the corresponding rows of $\mathbf{V}$ from Eq. (25) associated with the cable tensions included in ${ }^{a} \mathbf{t}^{s}$. The remaining $m-n$ columns of $\mathbf{W}$ and the corresponding cable tension vector along with the coordinates of the tension space vertices are denoted as ${ }^{b} \mathbf{W}^{s},{ }^{b} \mathbf{t}^{s}$ and ${ }^{b} \mathbf{V}^{s}$ respectively. Thus for the sth combination, the static equilibrium of the moving platform defined in Eq. (5) can be expressed by splitting the Wrench matrix into its square sub matrix ${ }^{a} \mathbf{W}^{s}$ and the remaining columns ${ }^{b} \mathbf{W}^{s}$ as

$$
{ }^{a} \mathbf{W}^{s}{ }^{a} \mathbf{t}^{s}+{ }^{b} \mathbf{W}^{s}{ }^{b} \mathbf{t}^{s}=\mathbf{w} \Longrightarrow{ }^{a} \mathbf{W}^{s a} \mathbf{t}^{s}=\mathbf{w}-{ }^{b} \mathbf{W}^{s}{ }^{b} \mathbf{t}^{s} \quad s=1, \ldots, C_{n}^{m}
$$

Therefore, the coordinates of the MCDPR tension space vertices in Eq. (25) can be expressed for the sth combination as,

$$
\mathbf{V}^{s}=\left[\begin{array}{l}
{ }^{a} \mathbf{V}^{s} \\
{ }^{b} \mathbf{V}^{s}
\end{array}\right]=\left[\begin{array}{lllll}
{ }^{a} \underline{\mathbf{v}}_{1}^{s} & \ldots & { }^{a} \underline{\mathbf{v}}_{l}^{s} & \ldots & { }^{a} \underline{\mathbf{v}}_{v}^{s} \\
{ }^{b} \underline{\mathbf{s}}_{1}^{s} & \ldots & \underline{\mathbf{v}}_{l}^{s} & \ldots & { }^{b} \underline{\mathbf{v}}_{v}^{s}
\end{array}\right]
$$

where ${ }^{a} \underline{\mathbf{v}}_{l}^{s}$ and ${ }^{b} \underline{\mathbf{v}}_{l}^{s}$ denote the $l$ th column of ${ }^{a} \mathbf{V}^{s}$ and ${ }^{b} \mathbf{V}^{s}$, respectively. Equation (36) amounts to a linear system of equations having $n$ number of equations with $n$ unknowns $\left({ }^{a} \mathbf{t}^{s}\right)$. Gaussian Elimination Algorithm [38] is used to solve such linear system of equations. The Algorithm determines the components of a cable tension vector ${ }^{a} \mathbf{t}^{s}$. The meaningful solutions i.e. the solutions for the cable tensions $t_{i j}$ included in ${ }^{a} \mathbf{t}^{s}$ only are extracted and used to map the static equilibrium conditions of $\mathscr{M}_{j}$ into the wrench space explained as follows.

Amongst the $m_{j}$ cables attached to $\mathscr{M}_{j}$, let $m_{j a}$ be the number of cables whose tensions are components of vector ${ }^{a} \mathbf{t}_{j}^{s}$. The tensions of the remaining $m_{j b}=m_{j}-m_{j a}$ cables are the components of vector ${ }^{b} \mathbf{t}_{j}^{s}$. As a consequence, the cable tension vector $\mathbf{t}_{j}$ and its associated actuation wrench matrix $\mathbf{W}_{j}$ defined in Eq. (7) can also be expressed as:

$$
\begin{gathered}
\mathbf{t}_{j}^{s}=\left[\begin{array}{ll}
a_{\mathbf{t}_{j}}{ }^{T} & b_{\mathbf{t}_{j}}^{\mathbf{s}_{j}}
\end{array}\right]^{T}=\left[{ }^{a} t_{1 j}^{s} \ldots{ }^{a} t_{o j}^{s} \ldots{ }^{a} t_{m_{j a j}}^{s}{ }^{b} t_{1 j}^{s} \ldots{ }^{b} t_{r j}^{s} \ldots{ }^{b} t_{m_{j b j} j}^{s}\right]^{T}, \\
\mathbf{W}_{j}^{s}=\left[{ }^{a} \mathbf{w}_{1 j}^{s} \ldots{ }^{a} \mathbf{w}_{o j}^{s} \ldots{ }^{a} \mathbf{w}_{m_{j a j}}^{s}{ }^{b} \mathbf{w}_{1 j}^{s} \ldots{ }^{b} \mathbf{w}_{r j}^{s} \ldots{ }^{b} \mathbf{w}_{m_{j b j}}^{s}\right],
\end{gathered}
$$

where $s=1, \ldots, C_{n}^{m} \cdot{ }^{a} t_{o j}^{s}, o=1, \ldots, m_{j a}$ and ${ }^{b} t_{r j}^{s}, r=1, \ldots, m_{j b}$ are the $o$ th and $r$ th components of ${ }^{a} t_{j}^{s}$ and ${ }^{b} \mathbf{t}_{j}^{s}$, respectively. ${ }^{a} \mathbf{w}_{o j}^{s}\left({ }^{b} \mathbf{w}_{r j}^{s}\right.$, resp.) is the actuation wrench associated with the cable tension ${ }^{a} t_{o j}^{s}\left({ }^{b} t_{r j}^{s}\right.$, resp.). Using Gaussian Elimination Algorithm, the cable tension ${ }^{a} t_{o j}^{s}, o=1, \ldots, m_{j a}$ is obtained as:

$$
a_{t_{o j}^{s}}=\frac{\alpha_{o j}^{s}\left(\mathbf{w}-{ }^{b} \mathbf{W}^{s} b^{s}\right)}{\alpha_{o j}^{s}{ }^{a} \mathbf{w}_{o j}^{s}}, \quad o=1, \ldots, m_{j a}, s=1, \ldots, C_{n}^{m}
$$

where $\alpha_{o j}^{s}$ is a $n$-dimensional row vector acquired from Gaussian Elimination Algorithm. The output of algorithm for the MCDPR under study is presented in Appendix A. From Eq. (39), the tensions in the cables attached to $\mathscr{M}_{j}$ are substituted in the corresponding static equilibrium condition to map it into the wrench space presented in the following sections. 


\subsubsection{Hyperplanes associated with the tipping conditions}

This section presents the hyperplanes of the AWS associated with the tipping conditions of $\mathscr{M}_{j}$ about the footprint boundary $\mathscr{L}_{C_{k j}}$, noted as $\mathscr{H}_{t k j}^{s}, k=1, \ldots, c_{j}, s=1, \ldots, C_{n}^{m}$. Using Eq. (38a), the tipping conditions of $\mathscr{M}_{j}$ defined by Eq. (12) about $\mathscr{L}_{C_{k j}}$ is expressed as:

$$
\begin{aligned}
& \mathbf{u}_{C_{k j}}^{T}\left(\left(\mathbf{g}_{j}-\mathbf{c}_{k j}\right) \times \mathbf{w}_{g j}\right)+\sum_{o=1}^{m_{j a}} \mathbf{u}_{C_{k j}}^{T}\left(\left(\mathbf{c}_{k j}-{ }^{a} \mathbf{b}_{o j}^{s}\right) \times{ }^{a} \mathbf{u}_{o j}^{s}\right){ }^{a} t_{o j}^{s} \\
& +\sum_{r=1}^{m_{j b}} \mathbf{u}_{C_{k j}}^{T}\left(\left(\mathbf{c}_{k j}-{ }^{b} \mathbf{b}_{r j}^{s}\right) \times{ }^{b} \mathbf{u}_{r j}^{s}\right){ }^{b} t_{r j}^{s} \leq 0, \quad k=1, \ldots, c_{j}, s=1, \ldots, C_{n}^{m} .
\end{aligned}
$$

where ${ }^{a} \mathbf{b}_{o j}^{s}$ and ${ }^{b} \mathbf{b}_{r j}^{s}$ are the coordinate vectors of the anchor points to which the cables of tension vectors ${ }^{a} \mathbf{t}_{o j}^{s}$ and ${ }^{b} \mathbf{t}_{r j}^{s}$ are attached. ${ }^{a} \mathbf{u}_{o j}^{s}$ and ${ }^{b} \mathbf{u}_{r j}^{s}$ are the directional vector of those cables. Substituting Eq. (39) in Eq. (40) yields,

$$
\begin{aligned}
& \sum_{o=1}^{m_{j a}} \frac{\mathbf{u}_{C_{k j}}^{T}\left(\left(\mathbf{c}_{k j}-{ }^{a} \mathbf{b}_{o j}^{s}\right) \times{ }^{a} \mathbf{u}_{o j}^{s}\right) \alpha_{o j}^{s}}{\alpha_{o j}^{s}{ }^{a} \mathbf{w}_{o j}^{s}}\left[\begin{array}{c}
\mathbf{f} \\
\mathbf{m}
\end{array}\right]-\sum_{o=1}^{m_{j a}} \frac{\mathbf{u}_{C_{k j}}^{T}\left(\left(\mathbf{c}_{k j}-{ }^{a} \mathbf{b}_{o j}^{s}\right) \times{ }^{a} \mathbf{u}_{o j}^{s}\right) \alpha_{o j}^{s}}{\alpha_{o j}^{s}{ }^{b} \mathbf{W}_{o j}^{s} b_{\mathbf{t}^{s}}^{s}} \\
& +\sum_{r=1}^{m_{j b}} \mathbf{u}_{C_{k j}}^{T}\left(\left(\mathbf{c}_{k j}-{ }^{b} \mathbf{b}_{r j}^{s}\right) \times{ }^{b} \mathbf{u}_{r j}^{s}\right){ }^{b} t_{r j}^{s}+\mathbf{u}_{C_{k j}}^{T}\left(\left(\mathbf{g}_{j}-\mathbf{c}_{k j}\right) \times \mathbf{w}_{g j}\right) \leq 0, k=1, \ldots, c_{j}, s=1, \ldots, C_{n}^{m} .
\end{aligned}
$$

Equation. (41) gives the tipping conditions for $\mathscr{M}_{j}$ expressed in the wrench space. Eq. (41) characterizes a hyperplane $\mathscr{H}_{t k j}^{s}$ :

$$
\mathscr{H}_{t k j}^{s}:\left(\mathbf{e}_{t k j}^{s}\right)^{T}\left[\begin{array}{c}
\mathbf{f} \\
\mathbf{m}
\end{array}\right] \leq d_{t k j}^{s}, k=1, \ldots, c_{j}, s=1, \ldots, C_{n}^{m},
$$

where $\mathbf{e}_{t k j}^{s}$ is a $n$-dimensional unit vector orthogonal to $\mathscr{H}_{t k j}^{s}$ expressed as:

$$
\mathbf{e}_{t k j}^{s}=\sum_{o=1}^{m_{j a}} \frac{\mathbf{u}_{C_{k j}}^{T}\left(\left(\mathbf{c}_{k j}-{ }^{a} \mathbf{b}_{o j}^{s}\right) \times{ }^{a} \mathbf{u}_{o j}^{s}\right)}{\alpha_{o j}^{s}{ }^{a} \mathbf{w}_{o j}^{s}} \alpha_{o j}^{s}, k=1, \ldots, c_{j}, s=1, \ldots, C_{n}^{m} .
$$

The distance from the origin of the wrench set to hyperplane $\mathscr{H}_{t k j}^{s}$ along $\mathbf{e}_{t k j}^{s}$ is defined by $d_{t k j}^{s}$. This distance is a function of the weight of $\mathscr{M}_{j}$ and the cable tensions included in ${ }^{b} \mathbf{t}^{s}$, namely,

$$
\begin{aligned}
d_{t k j}^{s}= & \max \left(\sum_{o=1}^{m_{j a}} \frac{\mathbf{u}_{C_{k j}}^{T}\left(\left(\mathbf{c}_{k j}-{ }^{a} \mathbf{b}_{o j}^{s}\right) \times{ }^{a} \mathbf{u}_{o j}^{s}\right) \alpha_{o j}^{s}}{\alpha_{o j}^{s}{ }^{b} \mathbf{w}_{o j}^{s}} \mathbf{W}^{s}{ }^{b} \underline{\mathbf{v}}_{l}^{s}, l=\{1, \ldots, v\}\right) \\
& -\min \left(\sum_{r=1}^{m_{j b}} \mathbf{u}_{C_{k j}}^{T}\left(\left(\mathbf{c}_{k j}-{ }^{b} \mathbf{b}_{r j}^{s}\right) \times{ }^{b} \mathbf{u}_{r j}^{s}\right){ }^{b} v_{r d j}^{s}, d=\left\{1, \ldots, v_{j}\right\}\right) \\
& -\mathbf{u}_{C_{k j}}^{T}\left(\left(\mathbf{g}_{j}-\mathbf{c}_{k j}\right) \times \mathbf{w}_{g j}\right) \leq 0, k=1, \ldots, c_{j}, s=1, \ldots, C_{n}^{m} .
\end{aligned}
$$

where ${ }^{b} v_{r d j}^{s}$ is the $r$ th component of the tension space generated by cable tensions ${ }^{b} t_{r j}^{s}, r=1, \ldots, m_{j b}$. The shifting distance $d_{t k j}^{s}$ represents the wrench capabilities of the moving platform against the corresponding tipping conditions about $\mathscr{L}_{C_{k j}}$ for $s$ th combination. ${ }^{b} \mathbf{W}^{s}{ }^{b} \mathbf{v}_{l}^{s}$ represents the wrenches applied by tension space vertices in ${ }^{b} \mathbf{V}^{s}$ along $\mathbf{e}_{t k j}^{s}$. This must be maximized to acquire the maximal wrench capabilities of the moving platform. On the contrary, the term $\mathbf{u}_{C_{k j}}^{T}\left(\left(\mathbf{c}_{k j}-{ }^{b} \mathbf{b}_{r j}^{s}\right) \times{ }^{b} \mathbf{u}_{r j}^{s}\right)^{b} t_{r j}^{s}$ contributes to the tipping of $\mathscr{M}_{j}$ due to the cable tensions ${ }^{b} t_{r j}^{s}, r=1, \ldots, m_{j b}$, and thus should be minimized. Each mobile base generates up to $c_{j} \times C_{n}^{m}$ possible hyperplanes corresponding to its tipping conditions.

\subsubsection{Hyperplanes associated with the sliding conditions}

This section describes the hyperplanes of the AWS associated with sliding conditions of mobile base $\mathscr{M}_{j}$, noted as $\mathscr{H}_{f g j}^{s}, g=1, \ldots, 4, s=1, \ldots, C_{n}^{m}$, respectively. The sliding conditions defined by Eq. (19) can be expressed using Eq. (38a) 


$$
\sum_{o=1}^{m_{j a}} \mathbf{E}_{f}{ }^{a} \mathbf{u}_{o j}^{s}{ }^{a} t_{o j}^{s}+\sum_{r=1}^{m_{j b}} \mathbf{E}_{f}^{b} \mathbf{u}_{r j}^{s}{ }^{b} t_{r j}^{s}-\mathbf{E}_{f} \mathbf{w}_{g j} \leq \mathbf{0}_{4}, s=1, \ldots, C_{n}^{m} .
$$

Substituting Eq. (39) in Eq. (45) yields:

$$
\begin{aligned}
& \sum_{o=1}^{m_{j a}} \frac{\mathbf{E}_{f}{ }^{a} \mathbf{u}_{o j}^{s} \alpha_{o j}^{s}}{\alpha_{o j}^{s}{ }^{a} \mathbf{w}_{o j}^{s}}\left[\begin{array}{c}
\mathbf{f} \\
\mathbf{m}
\end{array}\right]-\sum_{o=1}^{m_{j a}} \frac{\mathbf{E}_{f}{ }^{a} \mathbf{u}_{o j}^{s} \alpha_{o j}^{s}}{\alpha_{o j}^{s}{ }^{a} \mathbf{w}_{o j}^{s}}{ }^{b} \mathbf{W}^{s} b^{s}+\sum_{r=1}^{m_{j b}} \mathbf{E}_{f}{ }^{b} \mathbf{u}_{r j}^{s}{ }^{b} t_{r j}^{s} \\
& -\mathbf{E}_{f} \mathbf{w}_{g j} \leq \mathbf{0}_{4}, \quad s=1, \ldots, C_{n}^{m} .
\end{aligned}
$$

Equation (46) expresses the sliding conditions of $\mathscr{M}_{j}$ into the wrench space. The latter can be directly expressed in the form of a hyperplane $\mathscr{H}_{f g j}^{s}$ as,

$$
\mathscr{H}_{f g j}^{s}:\left(\mathbf{e}_{f g j}^{s}\right)^{T}\left[\begin{array}{c}
\mathbf{f} \\
\mathbf{m}
\end{array}\right] \leq d_{f g j}^{s}, s=1, \ldots, C_{n}^{m}, g=1, \ldots, 4
$$

where $\mathbf{e}_{f g j}^{s}$ is a $n$-dimensional unit vector orthogonal to $\mathscr{H}_{f g j}^{s}$ expressed as:

$$
\mathbf{e}_{f g j}^{s}=\sum_{o=1}^{m_{j a}} \frac{\mathbf{E}_{f}^{g}{ }^{a} \mathbf{u}_{o j}^{s} \alpha_{o j}^{s}}{\alpha_{o j}^{s} a \mathbf{w}_{o j}^{s}}, s=1, \ldots, C_{n}^{m}, g=1, \ldots, 4
$$

$\mathbf{E}_{f}^{g}$ denotes the $g$ th row of $\mathbf{E}_{f}$. The shifted distance of $\mathscr{H}_{f g j}^{s}$ is denoted as $d_{f g j}^{s}$ :

$$
\begin{aligned}
d_{f g j}^{s}= & \max \left(\sum_{o=1}^{m_{j a}} \frac{\left.\mathbf{E}_{f}^{a} \mathbf{u}_{o j}^{s} \alpha_{o j}^{s}{ }^{b} \mathbf{W}_{o j}^{s}{ }^{b} \underline{\mathbf{v}}_{l}^{s}, l=\{1, \ldots, v\}\right) \mathbf{E}_{f}^{g} \mathbf{w}_{g j}+\mathbf{E}_{f}^{g} \mathbf{w}_{g j}}{}\right. \\
& -\min \left(\sum_{r=1}^{m_{j b}} \mathbf{E}_{f}^{g}{ }^{b} \mathbf{u}_{r j}^{s}{ }^{b} v_{r d j}^{s}, d=\left\{1, \ldots, v_{j}\right\}\right), s=1, \ldots, C_{n}^{m}, g=1, \ldots, 4 .
\end{aligned}
$$

The shifting distance $d_{f g j}^{s}$ represents the wrench capabilities of the moving platform against the corresponding sliding conditions associated with the $g$ th boundary of the linearized friction pyramid of $\mathscr{M}_{j}$. It is noteworthy that each mobile base can generates up to $4 \times C_{n}^{m}$ possible hyperplanes corresponding to its sliding conditions.

\section{Wrench Feasible Workspace of MCDPRs}

The Wrench Feasible Workspace of a MCDPR is the set of platform poses that are wrench-feasible [39]. The movingplatform pose will be wrench-feasible if the set of required wrenches, named Required Wrench Set, denoted as $\mathscr{R}$ is inscribed in Available Wrench Set $\mathscr{A}$, namely,

$$
\mathscr{R} \subseteq \mathscr{A}
$$

The capacity margin index $w$ defined in $[40,41]$ can be used to calculate if a moving-platform pose is wrench-feasible or not. It is also known as a measure of the robustness of the equilibrium of the robot, expressed as,

$$
w=\min \left(\min w_{x, y}\right),
$$

where $w_{x, y}$ is the signed distance from the $x$ th vertex of $\mathscr{R}$ to the $y$ th facet of $\mathscr{A} . w_{x, y}$ is positive when the moving-platform pose is wrench feasible, negative otherwise.

In the following sections the wrench capabilities of three MCDPRs are outlined. The results are compared with the wrench capabilities of the classical CDPRs which only takes into account the constraints associated with the cable tension limits. 


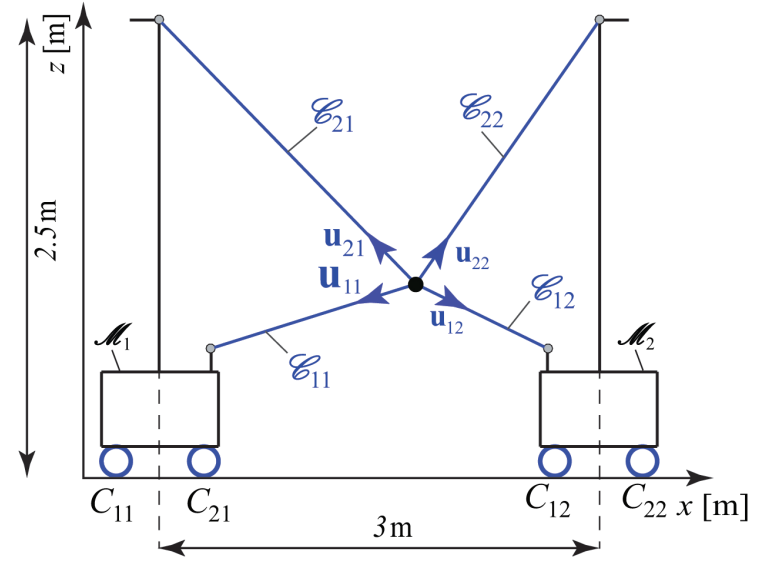

(a)

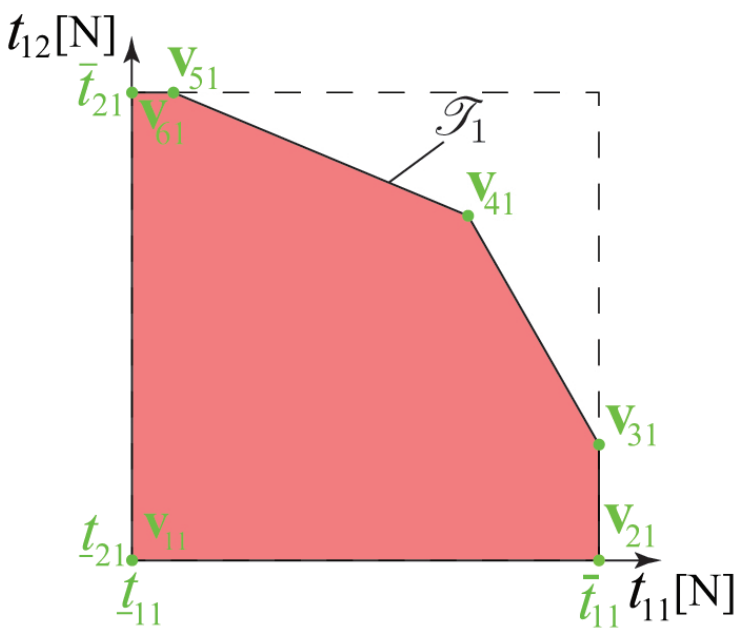

(c)

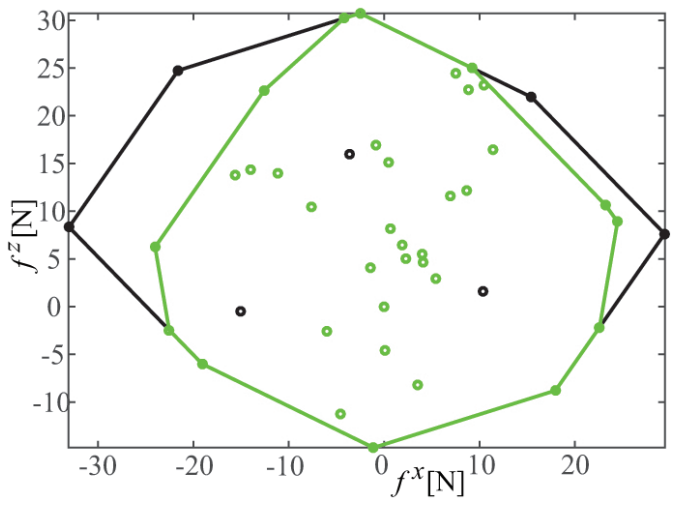

(e)

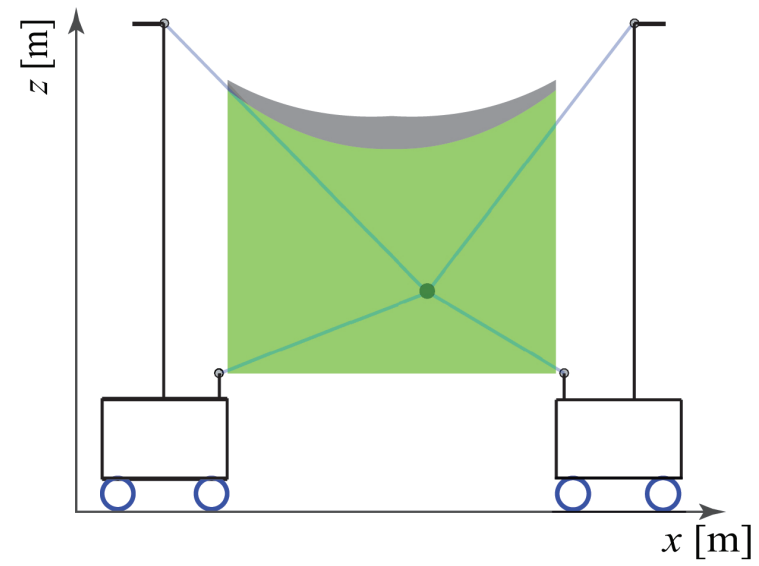

(b)

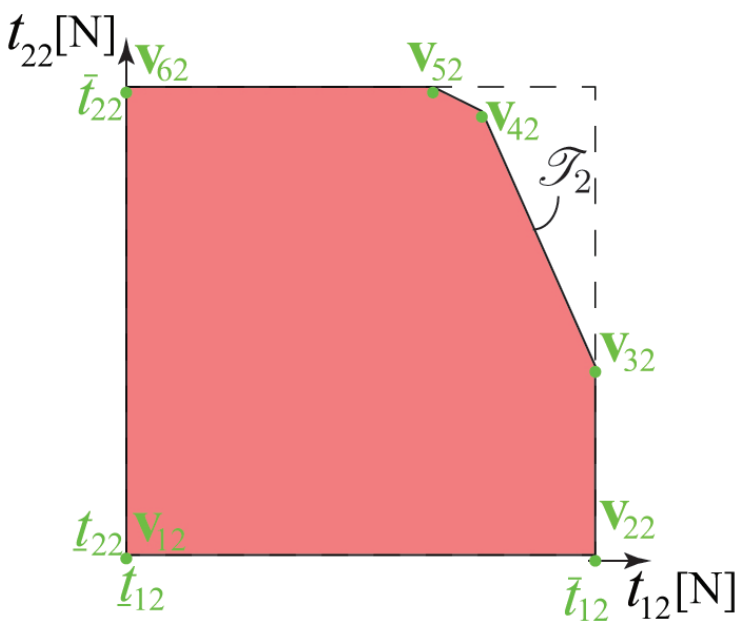

(d)

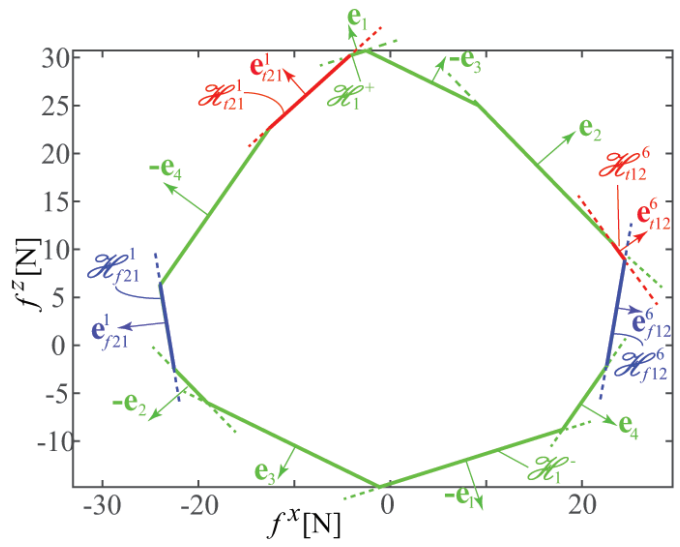

(f)

Fig. 6. (a) Planar MCDPR with one point-mass end-effector, two mobile bases and four cables (b) Static workspace (c,d) Modified cable tension space (e) $\mathscr{V}$-Representation of AWS of a CDPR (in black) and MCDPR (in green) (f) $\mathscr{H}$ - Representation of the AWS formed by the intersection of the hyperplanes

\subsection{Case study: $p=2, m=4$ and $n=2$ DoF MCDPR}

The first case study is a planar MCDPR with a $1.5 \mathrm{~kg}$ point mass end-effector shown in Fig. 6(a). The mass and size of each mobile base are equal to $13 \mathrm{~kg}$ and $0.7 \times 0.7 \times 0.71 \mathrm{~m}^{3}$, respectively. The corresponding cable tension space is illustrated in Figs. 6(c) and 6(d). The AWS defined by the Convex Hull approach is illustrated in Fig. 6(e). It can be observed that by considering only the cable tension limit constraints, the AWS in black corresponds to the wrench capabilities of a 
classical CDPR in the form of a zonotope. On the contrary, by taking into account the additional static equilibrium conditions associated with the mobile bases, the MCDPR AWS in green is no longer a zonotope, but a convex polytope.

Figure 6(f) depicts the AWS for the MCDPR configuration shown in Fig. 6(a), obtained by the HSM detailed in Sec. 4.2. The AWS due to cable tension limits is split by four additional hyperplanes due to the tipping and friction constraints named as $\mathscr{H}_{t 21}^{1}, \mathscr{H}_{f 21}^{1}, \mathscr{H}_{t 12}^{6}$ and $\mathscr{H}_{f 12}^{6}$ respectively. The hyperplane $\mathscr{H}_{t 21}^{1}\left(\mathscr{H}_{t 12}^{6}\right.$, resp. $)$ is associated with the tipping of $\mathscr{M}_{1}\left(\mathscr{M}_{2}\right.$, resp.) at $C_{21}\left(C_{12}\right.$, resp.) for $s=1\left(s=6\right.$, resp.), respectively. Similarly, the hyperplane $\mathscr{H}_{f 21}^{1}\left(\mathscr{H}_{f 12}^{6}\right.$, resp. $)$ is associated with the sliding of $\mathscr{M}_{1}\left(\mathscr{M}_{2}\right.$, resp.). The aforementioned hyperplanes are computed using ${ }^{a} \mathbf{t}^{s}$ and ${ }^{b} \mathbf{t}^{s}$ for $s=1$ and $s=6$, defined as:

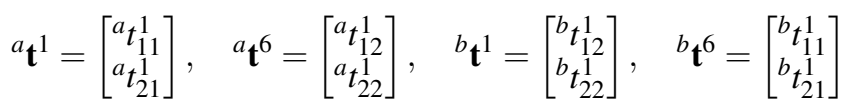

The static workspace of the planar MCDPR under study is shown in Fig. 6(b). The green region corresponds to the static workspace of the manipulator computed based on the cable tension limits, the mobile base tipping and sliding conditions. The gray region shows the area that cannot be reached by the point-mass end-effector because of mobile base tipping and/or sliding. The simulation and the experimental validations of this case study can be seen in video ${ }^{3}$.

\subsection{Case study: $p=4, m=8$ and $n=3$ DoF Planar MCDPR}

The previous case study is extended to a planar MCDPR with a $n=3$ DoF moving-platform illustrated in Fig. 7(a). The modified tension space is computed for a given moving-platform pose based on the cable tension limits, mobile base tipping and sliding conditions illustrated in Fig. 8. Based on the vertices of the tension space, the $\mathscr{V}$-representation of the AWS is shown in Fig. 7(b). The green area depicts the AWS of the manipulator characterized by the cable tension limits, the mobile base tipping and sliding conditions. The wrenches that cannot be generated by the end-effector due to the tipping and/or sliding of mobile bases are illustrated in gray.

Figure 9 depicts the hyperplanes associated with the static equilibrium of the MCDPR under study. The AWS due to cable tension limits is split by additional four hyperplanes due to the tipping and friction constraints named as $\mathscr{H}_{t 21}^{1}, \mathscr{H}_{t 21}^{2}$, $\mathscr{H}_{f 21}^{1}$ and $\mathscr{H}_{f 21}^{2}$ respectively. From Fig. 8, it appears that only the hyperplanes associated with the cable tension limits and the static equilibrium conditions of $\mathscr{M}_{1}$ define the facets of the AWS. The static equilibrium conditions of $\mathscr{M}_{2}$ do not affect $\mathscr{T}_{2}$, and thus do not affect the AWS.

\subsection{Case study: $p=2, m=8$ and $n=6$ DoF Spatial MCDPR}

The methodology described in Fig. 2 is used to trace the WFW of FASTKIT shown in Figs. 10(a) and 10(b) for different locations of the mobile bases. The green region corresponds to the static workspace of FASTKIT determined using the cable tension limits, the mobile base tipping and sliding conditions. The gray region illustrates the area that cannot be reached by the end-effector due to the tipping and/or sliding of the FASTKIT mobile bases. It should be noted that the higher the relative distance between the mobile bases, the smaller the wrench capability of FASTKIT along the vertical direction. The evolution of FASTKIT WFW as a function of the relative distance between the two mobile bases can be seen in video ${ }^{4}$.

\section{Conclusion}

In this paper, we have proposed a methodology to determine the Available Wrench Set of Mobile Cable-Driven Parallel Robots. The Available Wrench Set is required to trace the Wrench-Feasible Workspace of Mobile Cable-Driven Parallel Robots. The proposed workspace depends, not only on the Static Equilibrium of the moving-platform, but on the Static Equilibrium of the Mobile Bases. The Available Wrench Set is formed using two different approaches i.e. Convex Hull and the Hyperplane Shifting Method. The equivalence of both approaches were shown. Initially all the conditions associated with the Static Equilibrium of a Mobile Cable-Driven Parallel Robot are formulated. Compared to the classical Cable-Driven Parallel Robots, the additional Static Equilibrium conditions associated with the Mobile Bases may affect the Available Wrench Set. Multiple case studies are carried out in order to show that the approach is applicable to both planar and spatial Mobile Cable-Driven Parallel Robots. The proposed approach is experimentally validated on a Mobile Cable-Driven Parallel Robot with a point-mass end-effector and two Mobile Bases. Future work will focus on exploiting the proposed methodology in order to find the wrench-feasible poses during the trajectory planning of Mobile Cable-Driven Parallel Robots.

\footnotetext{
${ }^{3}$ https://youtu.be/UsvBnJ8q2v4

${ }^{4}$ https://youtu.be/EXbp1Bb7OCo
} 


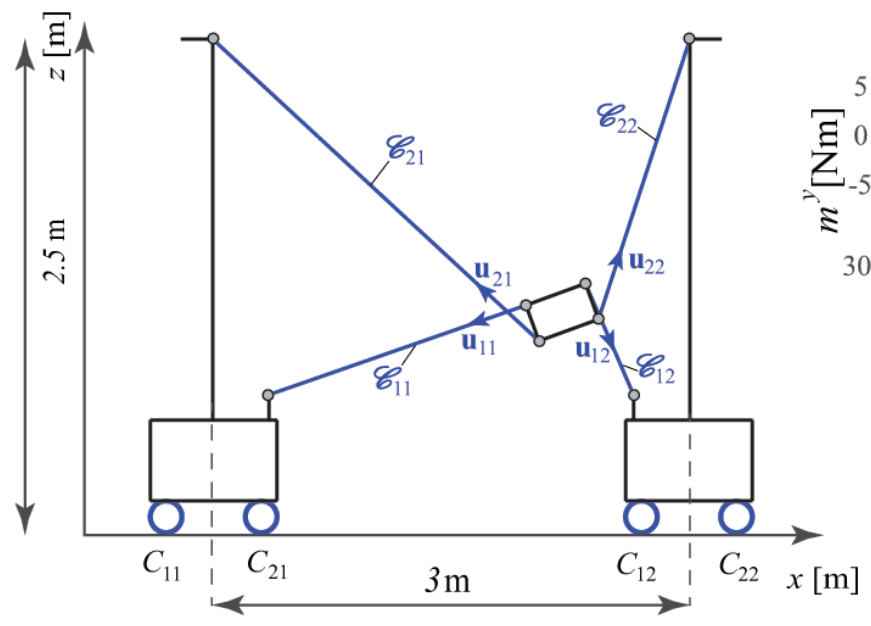

(a)

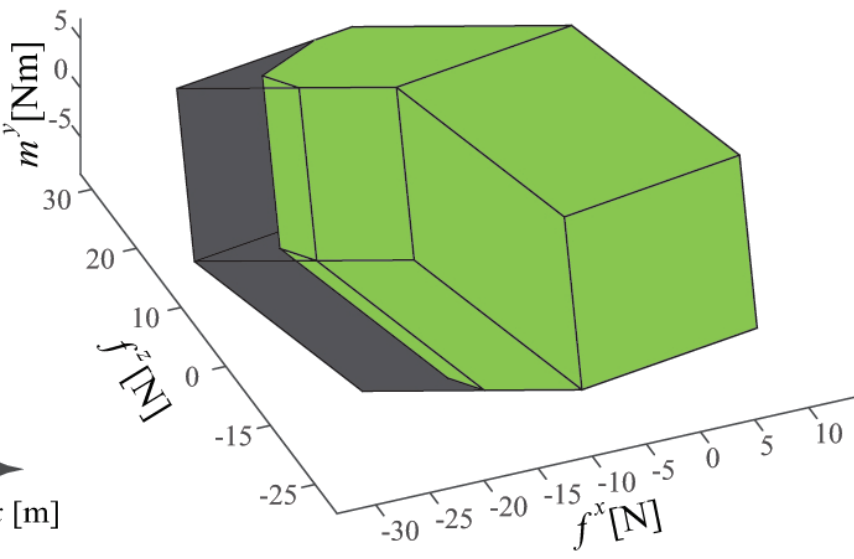

(b)

Fig. 7. (a) Configuration under study of $p=2, m=4$ and $n=3$ MCDPR (b) Comparison of AWS between CDPR (in black + green) and MCDPR (in green)

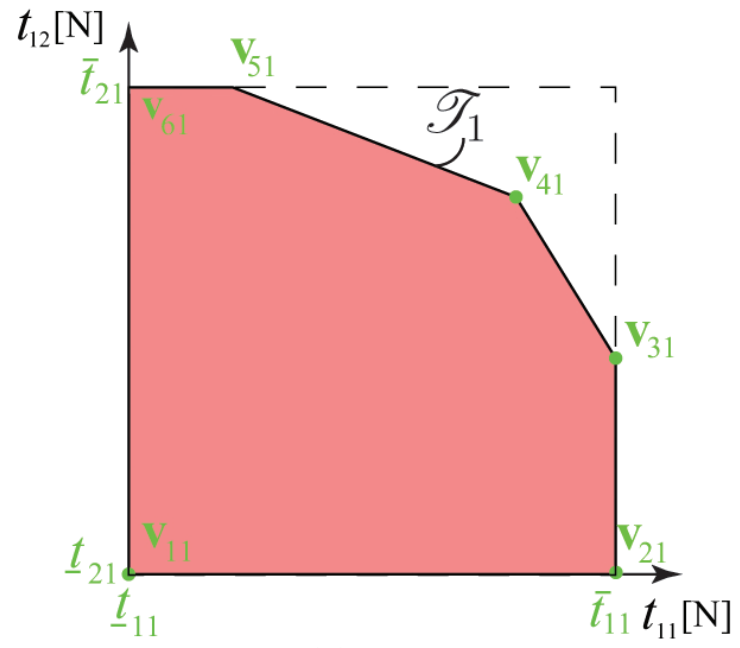

(a)

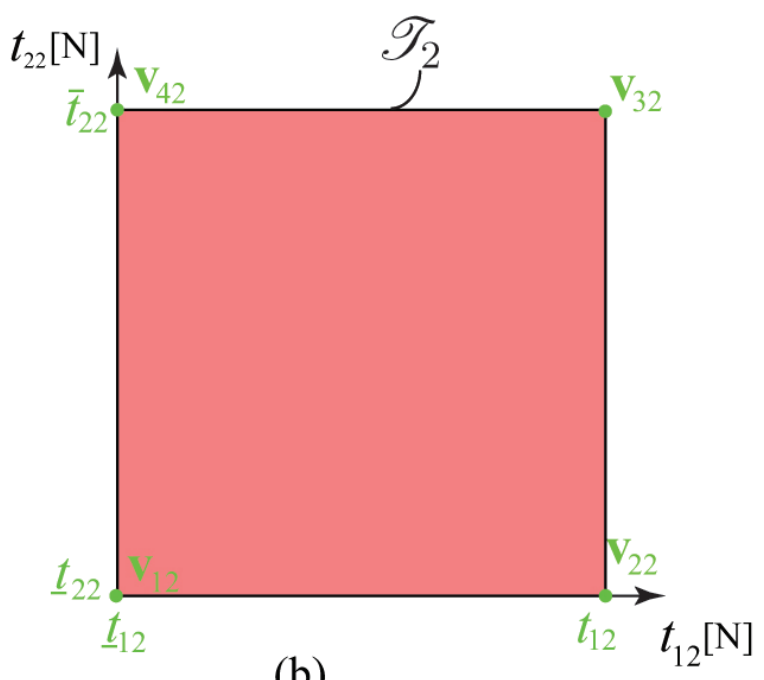

(b)

Fig. 8. (a) $\mathscr{T}_{1}$ and (b) $\mathscr{T}_{2}$ for MCDPR configuration in Fig. 7(a)

\section{Acknowledgments}

This research work was part of the European Project ECHORD++ "FASTKIT" dealing with the development of collaborative and Mobile Cable-Driven Parallel Robots for logistics. Moreover, Centrale Nantes is dutifully acknowledged for the doctorate thesis financial support provided to the first author of the paper.

\section{Nomenclature}

CDPRs : Cable-Driven Parallel Robots.

MCDPRs : Mobile Cable-Driven Parallel Robots.

DoF : Degree-of-Freedom.

AWS : Available Wrench Set.

WFW : Wrench Feasible Workspace.

ZMP : Zero-Moment Point.

$\mathscr{F}_{0}\left(O_{0}, x_{0}, y_{0}, z_{0}\right)$ : base frame centered at $O_{0}$.

$\mathscr{F}_{b j}\left(O_{b j}, x_{b j}, y_{b j}, z_{b j}\right):$ coordinate frame attached to the $j$ th Mobile Base.

$\mathscr{F}_{p}\left(O_{p}, x_{p}, y_{p}, z_{p}\right)$ : coordinate frame attached to the moving-platform.

$m$ : total number of cables carried by the MCDPR. 


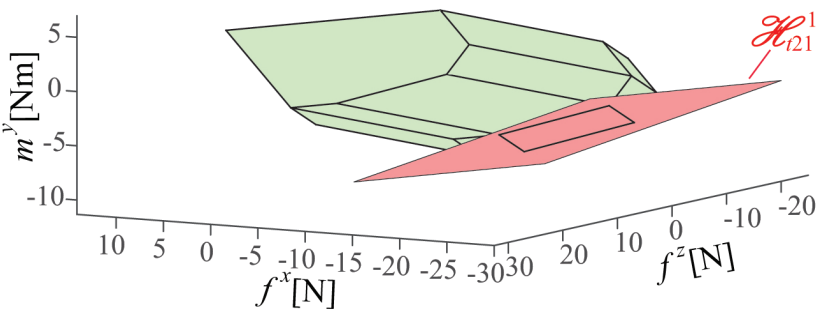

(a)

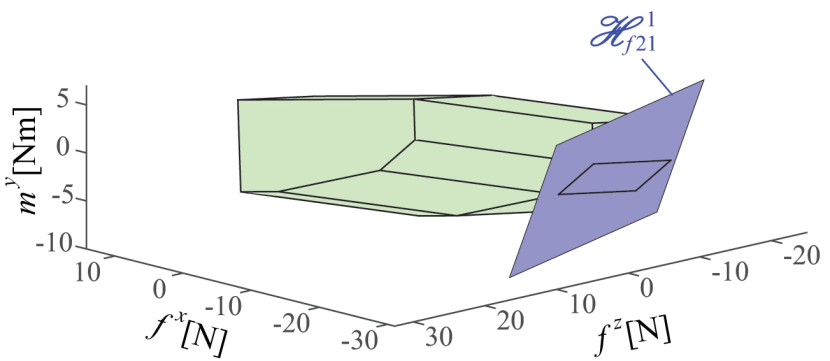

(c)

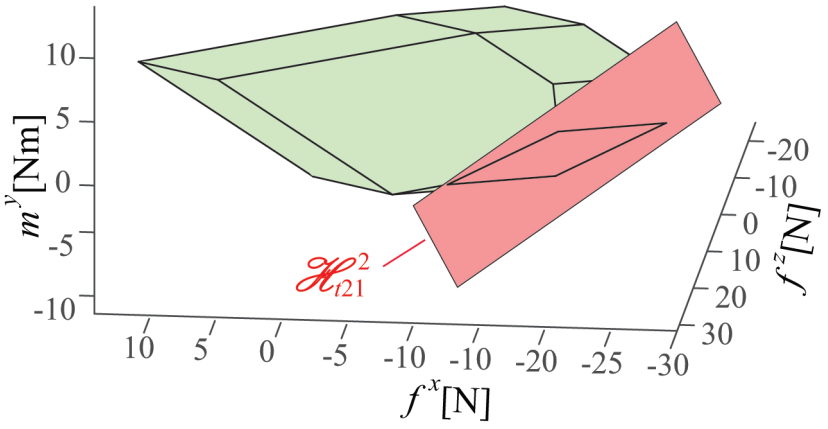

(b)

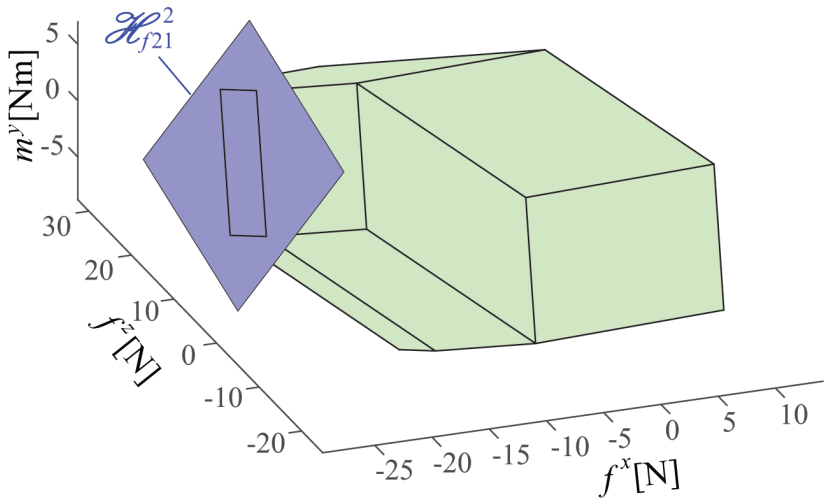

(d)

Fig. 9. Correspondence between the WFW facets obtained with the Convex Hull approach (in green) and those obtained with HSM: (a,b) Mobile Base tipping; (c,d) Mobile Base sliding for the moving-platform pose shown in Fig. 7(a)

$m_{j}$ : number of cables attached to the $j$ th mobile base.

$n$ : degree-of-freedom of the moving-platform.

$p:$ total number of mobile bases.

$\mathscr{M}_{j}: j$ th Mobile base.

$\mathscr{C}_{i j}: i$ th cable connected to the $j$ th mobile base.

$A_{i j}, B_{i j}$ : cable $\mathscr{C}_{i j}$ exit and anchor points depicted in Fig. 3.

$\mathscr{T}_{j}$ : tension space formed by the cables attached to the $j$ th mobile base.

$\mathbf{f}=\left[f^{x}, f^{y}, f^{z}\right]^{T}$ : forces applied by the cables onto the moving-platform.

$\mathbf{m}=\left[m^{x}, m^{y}, m^{z}\right]^{T}$ : moments applied by the cables onto the moving-platform.

$\mathbf{u}_{i j}$ : directional vector of cable $\mathscr{C}_{i j}$.

$\mathbf{c}_{r i j}$ : direction of the actuation moment applied by the cable $\mathscr{C}_{i j}$ onto the moving-platform.

$\mathbf{w}_{i j}=\left[\mathbf{u}_{i j}^{T}, \mathbf{c}_{r i j}^{T}\right]^{T}$ : actuation wrench generated by the cable $\mathscr{C}_{i j}$

$\mathbf{r}_{i j}$ : vector pointing from point $O_{P}$ to point $B_{i j}$.

$\mathbf{b}_{i j}$ : Cartesian coordinate vector of the platform attachment point $B_{i j}$

$t_{i j}:$ tension in the cable $\mathscr{C}_{i j}$

$\mathbf{W}_{j}$ : actuation wrenches exerted by the cables attached to $\mathscr{M}_{j}$ onto the moving-platform.

$\mathbf{w}$ : wrenches applied by the cables onto the moving-platform.

$\mathbf{t}_{j}$ : cable tension vector associated to the cables carried by $\mathscr{M}_{j}$.

$C_{k j}: k$ th wheel contact point of the $j$ th mobile base.

$\mathbf{c}_{k j}$ : Cartesian coordinate vector of point $C_{k j}$.

$\mathbf{f}_{c_{k j}}=\left[f_{c_{k j}}^{x}, f_{c_{k j}}^{y}, f_{c_{k j}}^{z}\right]$ : ground contact force at point $C_{k j}$.

$\mathscr{L}_{C_{k j}}$ : boundary of the $j$ th mobile base footprint between $C_{k j}$ and $C_{k+1 j}$.

$\mathbf{u}_{C_{k j}}$ : directional vector of line $\mathscr{L}_{C_{k j}}$ (see Fig.4(a)).

$m_{C_{k j}}$ : moment generated about $\mathscr{L}_{C_{k j}}$.

$\mathbf{g}_{j}$ : Cartesian coordinate vector of the $j$ th mobile base center of gravity.

$\mathbf{w}_{g j}$ : weight of $\mathscr{M}_{j}$.

$\mathbf{E}_{f}$ : Four sides of the friction pyramid

Corresponding author: Stéphane CARO, email: stephane.caro@1s2n.fr 

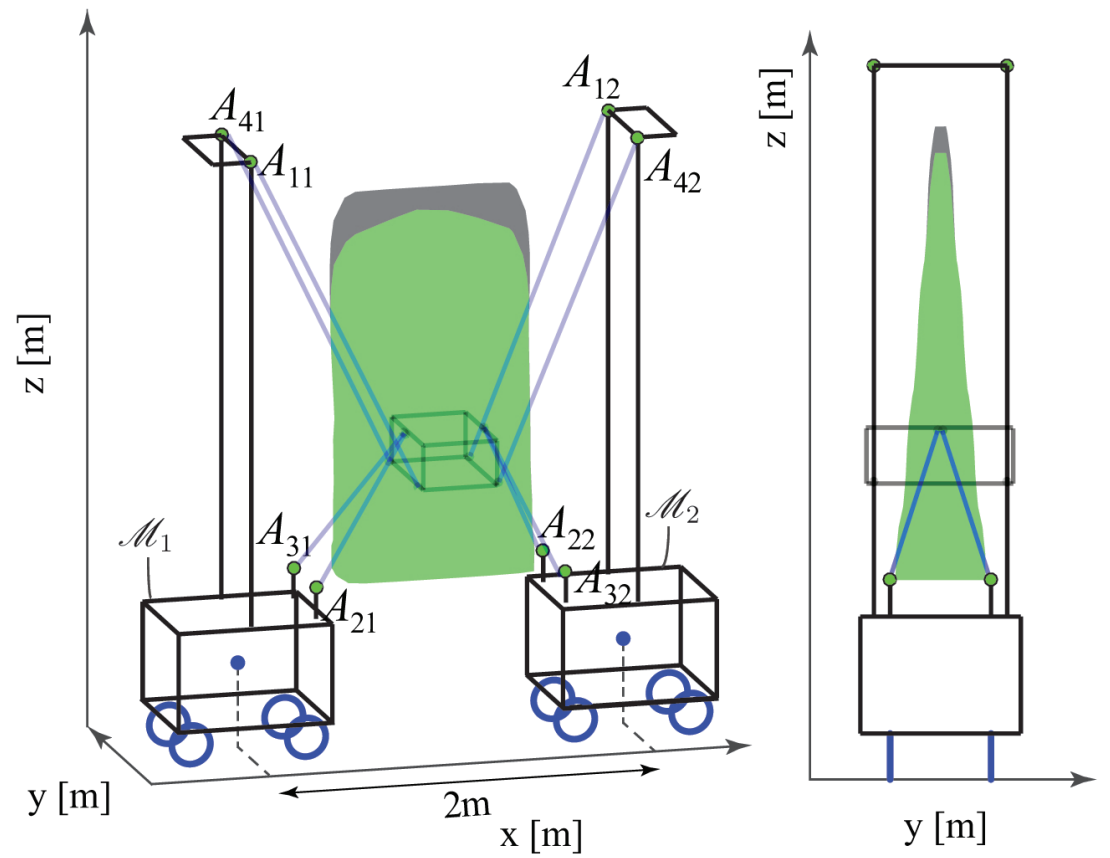

(a)

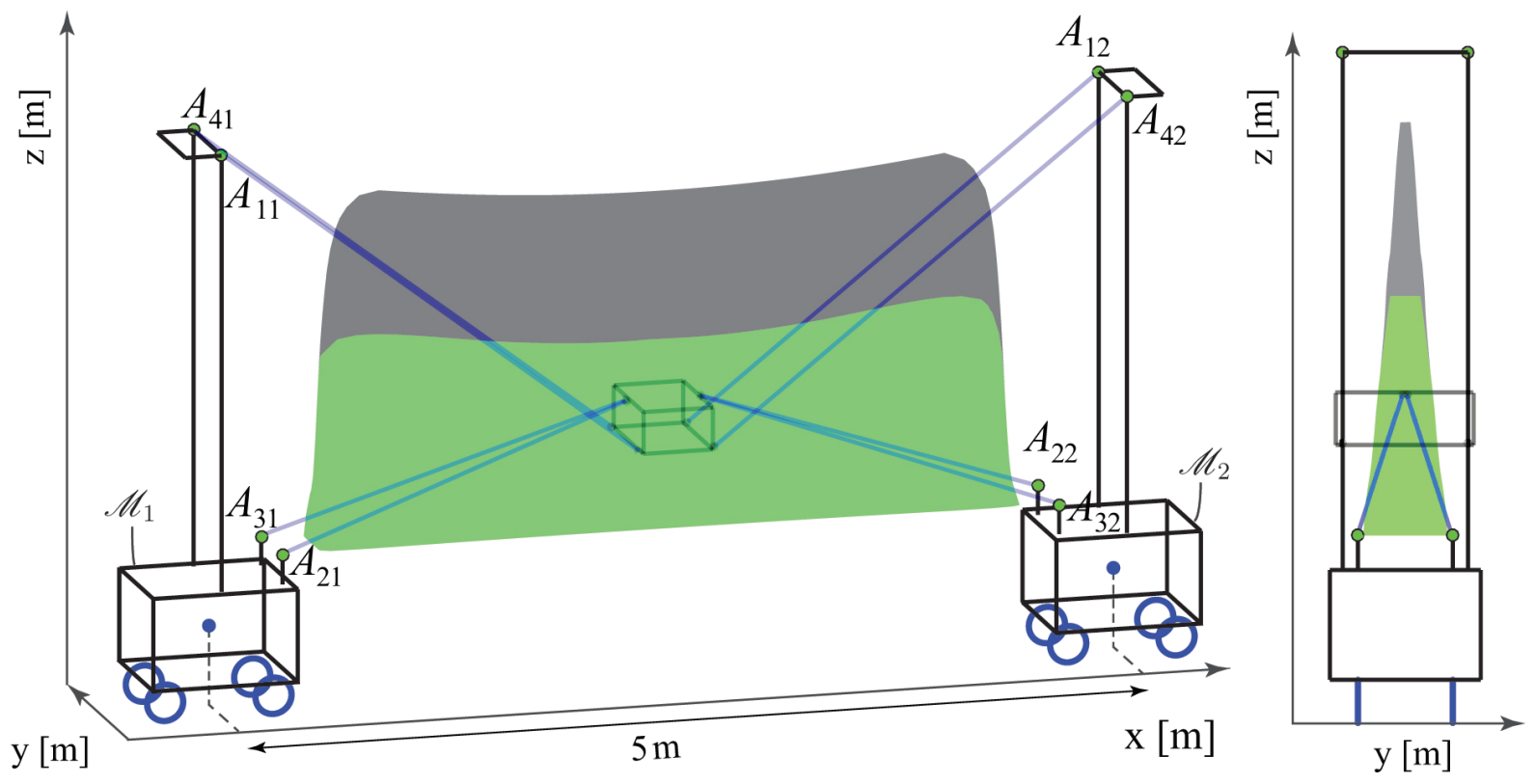

(b)

Fig. 10. WFW of FASTKIT at different configuration of mobile bases with a constant moving-platform orientation

${ }^{0} \mathbf{R}_{b j}$ : Rotation matrix from $\mathscr{F}_{0}$ to $\mathscr{F}_{b j}$.

$\mathscr{A}$ : Available Wrench Set of a Mobile Cable-Driven Parallel Robot.

$\mathscr{R}$ : Required Wrench Set.

$\mathscr{T}_{j}$ : tension Space formed by the cables mounted on $\mathscr{M}_{j}$.

$\mathbf{V}_{j}=\left[\mathbf{v}_{1 j} \mathbf{v}_{2 j} \ldots \mathbf{v}_{d j} \ldots \mathbf{v}_{v_{j}}\right]:$ coordinates of the vertices of $\mathscr{T}_{j}$.

$\mathbf{v}_{d j}$ : coordinate vector of the $d$ th vertex of $\mathscr{T}_{j}$.

$\mathscr{T}$ : MCDPR cable tension space.

$\mathbf{V}=\left[\underline{\mathbf{v}}_{1} \underline{\mathbf{v}}_{2} \underline{\mathbf{v}}_{l} \ldots \underline{\mathbf{v}}_{v}\right]:$ coordinates of the vertices of the MCDPR cable tension space.

$\underline{\mathbf{v}}_{l}$ : coordinates of the $l$ th vertex of the MCDPR cable tension space.

$\mathscr{H}_{q}^{+}, \mathscr{H}_{q}^{-}: q$ th pair of hyperplanes associated with the cable tension limits.

${ }^{a} \mathbf{W}^{s}: s t h n \times n$ sub-matrix of the wrench matrix $\mathbf{W}, s=1, \ldots, C_{n}^{m}$. 
${ }^{b} \mathbf{W}^{s}$ : those $m-n$ columns of $\mathbf{W}$ not located in ${ }^{a} \mathbf{W}^{s}$.

${ }^{a} \mathbf{t}^{s},{ }^{b} \mathbf{t}^{s}$ : cable tensions associated with the actuation wrenches of ${ }^{a} \mathbf{W}^{s},{ }^{b} \mathbf{W}^{s}$.

${ }^{a} \mathbf{V}^{s},{ }^{b} \mathbf{V}^{s}$ : coordinates of the tension space vertices of ${ }^{a} \mathbf{t}^{s},{ }^{b} \mathbf{t}^{s}$.

${ }^{a} \underline{\mathbf{v}}_{l}^{s},{ }^{b} \underline{\mathbf{v}}_{l}^{s}: l$ th column of ${ }^{a} \mathbf{V}^{s},{ }^{b} \mathbf{V}^{s}$.

$\mathbf{t}_{j}^{s}=\left[{ }^{a} \mathbf{t}_{j}^{s T},{ }^{b} \mathbf{t}_{j}^{s^{T}}\right]^{T}:$ sth division of the cable tensions $\mathbf{t}_{j}$ into ${ }^{a} \mathbf{t}_{j}^{s}$ and ${ }^{b} \mathbf{t}_{j}^{s}$.

${ }^{a} t_{o j}^{s}:$ oth component of ${ }^{a} \mathbf{t}_{j}^{s}$.

${ }^{b} t_{r j}^{s}: r$ th component of ${ }^{b} \mathbf{t}_{j}^{s}$.

${ }^{a} \mathbf{u}_{o j}^{s},{ }^{b} \mathbf{u}_{r j}^{s}$ : unit vectors of cables whose tension magnitudes are ${ }^{a} t_{o j}^{s},{ }^{b} t_{r j}^{s}$.

${ }^{a} \mathbf{b}_{o j}^{s},{ }^{b} \mathbf{b}_{r j}^{s}$ : Coordinate vectors of the anchor points to which the cables of tensions ${ }^{a} t_{o j}^{s},{ }^{b} t_{r j}^{s}$ are attached.

${ }^{a} \mathbf{w}_{o j}^{s},{ }^{b} \mathbf{w}_{r j}^{s}$ : actuation wrenches associated with the cable tensions ${ }^{a} t_{o j}^{s},{ }^{b} t_{r j}^{s}$.

$\mathscr{H}_{t k j}^{s}:$ sth hyperplane associated with the tipping of $\mathscr{M}_{j}$ about $\mathscr{L}_{C_{k j}}$.

$\mathscr{H}_{f g j}^{s}:$ sth hyperplane associated with the sliding of $\mathscr{M}_{j}$ in the direction normal to the $g$ th friction pyramid.

$\mathbf{e}_{t k j}^{s}, \mathbf{e}_{f g j}^{s}$ : unit vector orthogonal to its hyperplane $\mathscr{H}_{t k j}^{s}, \mathscr{H}_{f g j}^{s}$.

$d_{t k j}^{s}, d_{f g j}^{s}$ : shifting distances from the origin to the hyperplanes $\mathscr{H}_{t k j}^{s}, \mathscr{H}_{f g j}^{s}$, respectively.

\section{A Appendix A}

This appendix presents the output of the Gaussian Elimination Algorithm for the MCDPR under study in Fig. 3 composed of four mobile bases carrying eight cables named as $\mathscr{C}_{11}, \mathscr{C}_{21}, \mathscr{C}_{12}, \mathscr{C}_{22}, \mathscr{C}_{13}, \mathscr{C}_{23}, \mathscr{C}_{14}$ and $\mathscr{C}_{24}$ connected to a six DOF moving platform. Let for $s=1$, the wrench matrix $\mathbf{W}$ and the cable tension vector $\mathbf{t}$ are split as,

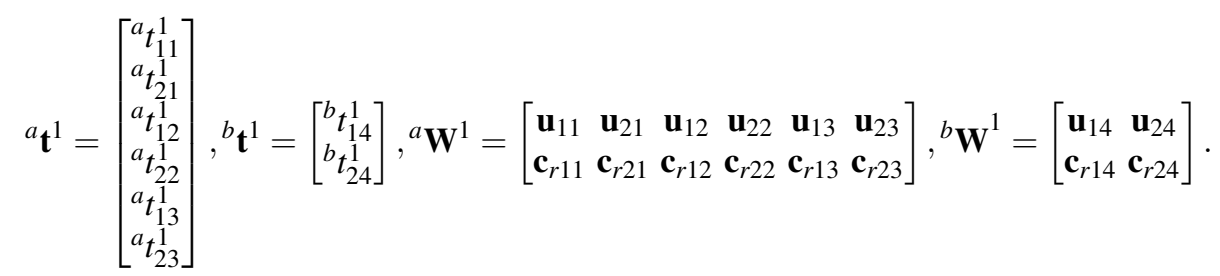

Using Gaussian Elimination Algorithm to solve for ${ }^{\mathbf{a}} \mathbf{t}^{1}$, its components are expressed as

$$
\begin{aligned}
& { }^{a} t_{11}^{1}=\frac{\alpha_{11}^{1}\left(\mathbf{w}-{ }^{b} \mathbf{W}^{s}{ }^{b} \mathbf{t}^{s}\right)}{\alpha_{11}^{1}\left[\mathbf{u}_{11}^{T} \mathbf{c}_{r 11}^{T}\right]^{T}}, \quad{ }^{a} t_{21}^{1}=\frac{\alpha_{21}^{1}\left(\mathbf{w}-{ }^{b} \mathbf{W}^{s}{ }^{b} \mathbf{t}^{s}\right)}{\alpha_{21}^{1}\left[\mathbf{u}_{21}^{T} \mathbf{c}_{r 21}^{T}\right]^{T}}, \quad{ }^{a} t_{12}^{1}=\frac{\alpha_{12}^{1}\left(\mathbf{w}-{ }^{b} \mathbf{W}^{s}{ }^{b} \mathbf{t}^{s}\right)}{\alpha_{12}^{1}\left[\mathbf{u}_{12}^{T} \mathbf{c}_{r 12}^{T}\right]^{T}}, \\
& { }^{a} t_{22}^{1}=\frac{\alpha_{22}^{1}\left(\mathbf{w}-{ }^{b} \mathbf{W}^{s} b_{\mathbf{t}^{s}}\right)}{\alpha_{22}^{1}\left[\mathbf{u}_{22}^{T} \mathbf{c}_{r 22}^{T}\right]^{T}}, \quad{ }^{a} t_{13}^{1}=\frac{\alpha_{13}^{1}\left(\mathbf{w}-{ }^{b} \mathbf{W}^{s}{ }^{b} \mathbf{t}^{s}\right)}{\alpha_{13}^{1}\left[\mathbf{u}_{13}^{T} \mathbf{c}_{r 13}^{T}\right]^{T}}, \quad{ }^{a} t_{23}^{1}=\frac{\alpha_{23}^{1}\left(\mathbf{w}-{ }^{b} \mathbf{W}^{s} b_{\mathbf{t}^{s}}\right)}{\alpha_{23}^{1}\left[\mathbf{u}_{23}^{T} \mathbf{c}_{r 23}^{T}\right]^{T}},
\end{aligned}
$$

where

$$
\alpha_{11}^{1}=\left[\begin{array}{cc}
{\left[\left(\left(\mathbf{c}_{r 13} \times \mathbf{c}_{r 23}\right)^{T} \mathbf{c}_{r 22}\right)\left(\mathbf{u}_{21} \times \mathbf{u}_{12}\right)^{+}\right.} & {\left[-\left(\left(\mathbf{u}_{21} \times \mathbf{u}_{12}\right)^{T} \mathbf{u}_{22}\right)\left(\mathbf{c}_{r 13} \times \mathbf{c}_{r 23}\right)-\right.} \\
\left(\left(\mathbf{c}_{r 12} \times \mathbf{c}_{r 23}\right)^{T} \mathbf{c}_{r 13}\left(\mathbf{u}_{21} \times \mathbf{u}_{22}\right)+\right. & \left(\left(\mathbf{u}_{21} \times \mathbf{u}_{22}\right)^{T} \mathbf{u}_{13}\right)\left(\mathbf{c}_{r 12} \times \mathbf{c}_{r 23}\right)- \\
\left(\left(\mathbf{c}_{r 12} \times \mathbf{c}_{r 22}\right)^{T} \mathbf{c}_{r 23}\right)\left(\mathbf{u}_{21} \times \mathbf{u}_{13}\right)+ & \left(\left(\mathbf{u}_{21} \times \mathbf{u}_{13}\right)^{T} \mathbf{u}_{23}\right)\left(\mathbf{c}_{r 12} \times \mathbf{c}_{r 22}\right)- \\
\left(\left(\mathbf{c}_{r 12} \times \mathbf{c}_{r 13}\right)^{T} \mathbf{c}_{r 22}\right)\left(\mathbf{u}_{21} \times \mathbf{u}_{23}\right)+ & \left(\left(\mathbf{u}_{21} \times \mathbf{u}_{23}\right)^{T} \mathbf{u}_{22}\right)\left(\mathbf{c}_{r 12} \times \mathbf{c}_{r 13}\right)- \\
\left(\left(\mathbf{c}_{r 21} \times \mathbf{c}_{r 23}\right)^{T} \mathbf{c}_{r 13}\right)\left(\mathbf{u}_{12} \times \mathbf{u}_{22}\right)+ & \left(\left(\mathbf{u}_{12} \times \mathbf{u}_{22}\right)^{T} \mathbf{u}_{13}\right)\left(\mathbf{c}_{r 21} \times \mathbf{c}_{r 23}\right)- \\
\left(\left(\mathbf{c}_{r 23} \times \mathbf{c}_{r 22}\right)^{T} \mathbf{c}_{r 21}\right)\left(\mathbf{u}_{12} \times \mathbf{u}_{13}\right)+ & \left(\left(\mathbf{u}_{12} \times \mathbf{u}_{13}\right)^{T} \mathbf{u}_{21}\right)\left(\mathbf{c}_{r 23} \times \mathbf{c}_{r 22}\right)- \\
\left(\left(\mathbf{c}_{r 22} \times \mathbf{c}_{r 13}\right)^{T} \mathbf{c}_{r 21}\right)\left(\mathbf{u}_{12} \times \mathbf{u}_{23}\right)+ & \left(\left(\mathbf{u}_{12} \times \mathbf{u}_{23}\right)^{T} \mathbf{u}_{21}\right)\left(\mathbf{c}_{r 22} \times \mathbf{c}_{r 13}\right)- \\
\left(\left(\mathbf{c}_{r 21} \times \mathbf{c}_{r 12}\right)^{T} \mathbf{c}_{r 23}\right)\left(\mathbf{u}_{22} \times \mathbf{u}_{13}\right)+ & \left(\left(\mathbf{u}_{22} \times \mathbf{u}_{13}\right)^{T} \mathbf{u}_{23}\right)\left(\mathbf{c}_{r 21} \times \mathbf{c}_{r 12}\right)- \\
\left(\left(\mathbf{c}_{r 21} \times \mathbf{c}_{r 13}\right)^{T} \mathbf{c}_{r 12}\right)\left(\mathbf{u}_{22} \times \mathbf{u}_{23}\right)+ & \left(\left(\mathbf{u}_{22} \times \mathbf{u}_{23}\right)^{T} \mathbf{u}_{12}\right)\left(\mathbf{c}_{r 21} \times \mathbf{c}_{r 13}\right)- \\
\left.\left(\left(\mathbf{c}_{r 22} \times \mathbf{c}_{r 21}\right)^{T} \mathbf{c}_{r 12}\right)\left(\mathbf{u}_{13} \times \mathbf{u}_{23}\right)\right]^{T} & \left.\left(\left(\mathbf{u}_{13} \times \mathbf{u}_{23}\right)^{T} \mathbf{u}_{12}\right)\left(\mathbf{c}_{r 22} \times \mathbf{c}_{r 21}\right)\right]^{T}
\end{array}\right]
$$

Corresponding author: Stéphane CARO, email: stephane.caro@1s2n.fr 


$$
\alpha_{21}^{1}=\left[\begin{array}{cc}
{\left[\left(\left(\mathbf{c}_{r 13} \times \mathbf{c}_{r 23}\right)^{T} \mathbf{c}_{r 22}\right)\left(\mathbf{u}_{11} \times \mathbf{u}_{12}\right)+\left[-\left(\left(\mathbf{u}_{11} \times \mathbf{u}_{12}\right)^{T} \mathbf{u}_{22}\right)\left(\mathbf{c}_{r 13} \times \mathbf{c}_{r 23}\right)-\right.\right.} \\
\left(\left(\mathbf{c}_{r 12} \times \mathbf{c}_{r 23}\right)^{T} \mathbf{c}_{r 13}\right)\left(\mathbf{u}_{11} \times \mathbf{u}_{22}\right)+ & \left(\left(\mathbf{u}_{11} \times \mathbf{u}_{22}\right)^{T} \mathbf{u}_{13}\right)\left(\mathbf{c}_{r 12} \times \mathbf{c}_{r 23}\right)^{-} \\
\left(\left(\mathbf{c}_{r 12} \times \mathbf{c}_{r 22}\right)^{T} \mathbf{c}_{r 23}\right)\left(\mathbf{u}_{11} \times \mathbf{u}_{13}\right)+ & \left(\left(\mathbf{u}_{11} \times \mathbf{u}_{13}\right)^{T} \mathbf{u}_{23}\right)\left(\mathbf{c}_{r 12} \times \mathbf{c}_{r 22}\right)- \\
\left(\left(\mathbf{c}_{r 12} \times \mathbf{c}_{r 13}\right)^{T} \mathbf{c}_{r 22}\right)\left(\mathbf{u}_{11} \times \mathbf{u}_{23}\right)+ & \left(\left(\mathbf{u}_{11} \times \mathbf{u}_{23}\right)^{T} \mathbf{u}_{22}\right)\left(\mathbf{c}_{r 12} \times \mathbf{c}_{r 13}\right)- \\
\left(\left(\mathbf{c}_{r 11} \times \mathbf{c}_{r 23}\right)^{T} \mathbf{c}_{r 13}\right)\left(\mathbf{u}_{12} \times \mathbf{u}_{22}\right)+ & \left(\left(\mathbf{u}_{12} \times \mathbf{u}_{22}\right)^{T} \mathbf{u}_{13}\right)\left(\mathbf{c}_{r 11} \times \mathbf{c}_{r 23}\right)- \\
\left(\left(\mathbf{c}_{r 23} \times \mathbf{c}_{r 22}\right)^{T} \mathbf{c}_{r 11}\right)\left(\mathbf{u}_{12} \times \mathbf{u}_{13}\right)+ & \left(\left(\mathbf{u}_{12} \times \mathbf{u}_{13}\right)^{T} \mathbf{u}_{11}\right)\left(\mathbf{c}_{r 23} \times \mathbf{c}_{r 22}\right)- \\
\left(\left(\mathbf{c}_{r 22} \times \mathbf{c}_{r 13}\right)^{T} \mathbf{c}_{r 11}\right)\left(\mathbf{u}_{12} \times \mathbf{u}_{23}\right)+ & \left(\left(\mathbf{u}_{12} \times \mathbf{u}_{23}\right)^{T} \mathbf{u}_{11}\right)\left(\mathbf{c}_{r 22} \times \mathbf{c}_{r 13}\right)- \\
\left(\left(\mathbf{c}_{r 11} \times \mathbf{c}_{r 12}\right)^{T} \mathbf{c}_{r 23}\right)\left(\mathbf{u}_{22} \times \mathbf{u}_{13}\right)+ & \left(\left(\mathbf{u}_{22} \times \mathbf{u}_{13}\right)^{T} \mathbf{u}_{23}\right)\left(\mathbf{c}_{r 11} \times \mathbf{c}_{r 12}\right)- \\
\left(\left(\mathbf{c}_{r 11} \times \mathbf{c}_{r 13}\right)^{T} \mathbf{c}_{r 12}\right)\left(\mathbf{u}_{22} \times \mathbf{u}_{23}\right)+ & \left(\left(\mathbf{u}_{22} \times \mathbf{u}_{23}\right)^{T} \mathbf{u}_{12}\right)\left(\mathbf{c}_{r 11} \times \mathbf{c}_{r 13}\right)- \\
\left.\left(\left(\mathbf{c}_{r 22} \times \mathbf{c}_{r 11}\right)^{T} \mathbf{c}_{r 12}\right)\left(\mathbf{u}_{13} \times \mathbf{u}_{23}\right)\right]^{T} & \left.\left(\left(\mathbf{u}_{13} \times \mathbf{u}_{23}\right)^{T} \mathbf{u}_{12}\right)\left(\mathbf{c}_{r 22} \times \mathbf{c}_{r 11}\right)\right]^{T}
\end{array}\right]
$$

$$
\alpha_{12}^{1}=\left[\begin{array}{cc}
{\left[\left(\left(\mathbf{c}_{r 13} \times \mathbf{c}_{r 23}\right)^{T} \mathbf{c}_{r 22}\right)\left(\mathbf{u}_{11} \times \mathbf{u}_{21}\right)+\right.} & {\left[-\left(\left(\mathbf{u}_{11} \times \mathbf{u}_{21}\right)^{T} \mathbf{u}_{22}\right)\left(\mathbf{c}_{r 13} \times \mathbf{c}_{r 23}\right)-\right.} \\
\left(\left(\mathbf{c}_{r 21} \times \mathbf{c}_{r 23}\right)^{T} \mathbf{c}_{r 13}\right)\left(\mathbf{u}_{11} \times \mathbf{u}_{22}\right)+ & \left(\left(\mathbf{u}_{11} \times \mathbf{u}_{22}\right)^{T} \mathbf{u}_{13}\right)\left(\mathbf{c}_{r 21} \times \mathbf{c}_{r 23}\right)- \\
\left(\left(\mathbf{c}_{r 21} \times \mathbf{c}_{r 22}\right)^{T} \mathbf{c}_{r 23}\right)\left(\mathbf{u}_{11} \times \mathbf{u}_{13}\right)+ & \left(\left(\mathbf{u}_{11} \times \mathbf{u}_{13}\right)^{T} \mathbf{u}_{23}\right)\left(\mathbf{c}_{r 21} \times \mathbf{c}_{r 22}\right)- \\
\left(\left(\mathbf{c}_{r 21} \times \mathbf{c}_{r 13}\right)^{T} \mathbf{c}_{r 22}\right)\left(\mathbf{u}_{11} \times \mathbf{u}_{23}\right)+ & \left(\left(\mathbf{u}_{11} \times \mathbf{u}_{23}\right)^{T} \mathbf{u}_{22}\right)\left(\mathbf{c}_{r 21} \times \mathbf{c}_{r 13}\right)- \\
\left(\left(\mathbf{c}_{r 11} \times \mathbf{c}_{r 23}\right)^{T} \mathbf{c}_{r 13}\right)\left(\mathbf{u}_{21} \times \mathbf{u}_{22}\right)+ & \left(\left(\mathbf{u}_{21} \times \mathbf{u}_{22}\right)^{T} \mathbf{u}_{13}\right)\left(\mathbf{c}_{r 11} \times \mathbf{c}_{r 23}\right)- \\
\left(\left(\mathbf{c}_{r 23} \times \mathbf{c}_{r 22}\right)^{T} \mathbf{c}_{r 11}\right)\left(\mathbf{u}_{21} \times \mathbf{u}_{13}\right)+ & \left(\left(\mathbf{u}_{21} \times \mathbf{u}_{13}\right)^{T} \mathbf{u}_{11}\right)\left(\mathbf{c}_{r 23} \times \mathbf{c}_{r 22}\right)- \\
\left(\left(\mathbf{c}_{r 22} \times \mathbf{c}_{r 13}\right)^{T} \mathbf{c}_{r 11}\right)\left(\mathbf{u}_{21} \times \mathbf{u}_{23}\right)+ & \left(\left(\mathbf{u}_{21} \times \mathbf{u}_{23}\right)^{T} \mathbf{u}_{11}\right)\left(\mathbf{c}_{r 22} \times \mathbf{c}_{r 13}\right)- \\
\left(\left(\mathbf{c}_{r 11} \times \mathbf{c}_{r 21}\right)^{T} \mathbf{c}_{r 23}\right)\left(\mathbf{u}_{22} \times \mathbf{u}_{13}\right)+ & \left(\left(\mathbf{u}_{22} \times \mathbf{u}_{13}\right)^{T} \mathbf{u}_{23}\right)\left(\mathbf{c}_{r 11} \times \mathbf{c}_{r 21}\right)- \\
\left(\left(\mathbf{c}_{r 11} \times \mathbf{c}_{r 13}\right)^{T} \mathbf{c}_{r 21}\right)\left(\mathbf{u}_{22} \times \mathbf{u}_{23}\right)+ & \left(\left(\mathbf{u}_{22} \times \mathbf{u}_{23}\right)^{T} \mathbf{u}_{21}\right)\left(\mathbf{c}_{r 11} \times \mathbf{c}_{r 13}\right)- \\
\left.\left(\left(\mathbf{c}_{r 22} \times \mathbf{c}_{r 11}\right)^{T} \mathbf{c}_{r 21}\right)\left(\mathbf{u}_{13} \times \mathbf{u}_{23}\right)\right]^{T} & \left.\left(\left(\mathbf{u}_{13} \times \mathbf{u}_{23}\right)^{T} \mathbf{u}_{21}\right)\left(\mathbf{c}_{r 22} \times \mathbf{c}_{r 11}\right)\right]^{T}
\end{array}\right]
$$

$$
\alpha_{22}^{1}=\left[\begin{array}{cc}
{\left[\left(\left(\mathbf{c}_{r 13} \times \mathbf{c}_{r 23}\right)^{T} \mathbf{c}_{r 12}\right)\left(\mathbf{u}_{11} \times \mathbf{u}_{21}\right)+\left[-\left(\left(\mathbf{u}_{11} \times \mathbf{u}_{21}\right)^{T} \mathbf{u}_{12}\right)\left(\mathbf{c}_{r 13} \times \mathbf{c}_{r 23}\right)-\right.\right.} \\
\left(\left(\mathbf{c}_{r 21} \times \mathbf{c}_{r 23}\right)^{T} \mathbf{c}_{r 13}\right)\left(\mathbf{u}_{11} \times \mathbf{u}_{12}\right)+ & \left(\left(\mathbf{u}_{11} \times \mathbf{u}_{12}\right)^{T} \mathbf{u}_{13}\right)\left(\mathbf{c}_{r 21} \times \mathbf{c}_{r 23}\right)- \\
\left(\left(\mathbf{c}_{r 21} \times \mathbf{c}_{r 12}\right)^{T} \mathbf{c}_{r 23}\right)\left(\mathbf{u}_{11} \times \mathbf{u}_{13}\right)+ & \left(\left(\mathbf{u}_{11} \times \mathbf{u}_{13}\right)^{T} \mathbf{u}_{23}\right)\left(\mathbf{c}_{r 21} \times \mathbf{c}_{r 12}\right)- \\
\left(\left(\mathbf{c}_{r 21} \times \mathbf{c}_{r 13}\right)^{T} \mathbf{c}_{r 12}\right)\left(\mathbf{u}_{11} \times \mathbf{u}_{23}\right)+ & \left(\left(\mathbf{u}_{11} \times \mathbf{u}_{23}\right)^{T} \mathbf{u}_{12}\right)\left(\mathbf{c}_{r 21} \times \mathbf{c}_{r 13}\right)- \\
\left(\left(\mathbf{c}_{r 11} \times \mathbf{c}_{r 23}\right)^{T} \mathbf{c}_{r 13}\right)\left(\mathbf{u}_{21} \times \mathbf{u}_{12}\right)+ & \left(\left(\mathbf{u}_{21} \times \mathbf{u}_{12}\right)^{T} \mathbf{u}_{13}\right)\left(\mathbf{c}_{r 11} \times \mathbf{c}_{r 23}\right)- \\
\left(\left(\mathbf{c}_{r 23} \times \mathbf{c}_{r 12}\right)^{T} \mathbf{c}_{r 11}\right)\left(\mathbf{u}_{21} \times \mathbf{u}_{13}\right)+ & \left(\left(\mathbf{u}_{21} \times \mathbf{u}_{13}\right)^{T} \mathbf{u}_{11}\right)\left(\mathbf{c}_{r 23} \times \mathbf{c}_{r 12}\right)- \\
\left(\left(\mathbf{c}_{r 12} \times \mathbf{c}_{r 13}\right)^{T} \mathbf{c}_{r 11}\right)\left(\mathbf{u}_{21} \times \mathbf{u}_{23}\right)+ & \left(\left(\mathbf{u}_{21} \times \mathbf{u}_{23}\right)^{T} \mathbf{u}_{11}\right)\left(\mathbf{c}_{r 12} \times \mathbf{c}_{r 13}\right)- \\
\left(\left(\mathbf{c}_{r 11} \times \mathbf{c}_{r 21}\right)^{T} \mathbf{c}_{r 23}\right)\left(\mathbf{u}_{12} \times \mathbf{u}_{13}\right)+ & \left(\left(\mathbf{u}_{12} \times \mathbf{u}_{13}\right)^{T} \mathbf{u}_{23}\right)\left(\mathbf{c}_{r 11} \times \mathbf{c}_{r 21}\right)- \\
\left(\left(\mathbf{c}_{r 11} \times \mathbf{c}_{r 13}\right)^{T} \mathbf{c}_{r 21}\right)\left(\mathbf{u}_{12} \times \mathbf{u}_{23}\right)+ & \left(\left(\mathbf{u}_{12} \times \mathbf{u}_{23}\right)^{T} \mathbf{u}_{21}\right)\left(\mathbf{c}_{r 11} \times \mathbf{c}_{r 13}\right)- \\
\left.\left(\left(\mathbf{c}_{r 12} \times \mathbf{c}_{r 11}\right)^{T} \mathbf{c}_{r 21}\right)\left(\mathbf{u}_{13} \times \mathbf{u}_{23}\right)\right]^{T} & \left.\left(\left(\mathbf{u}_{13} \times \mathbf{u}_{23}\right)^{T} \mathbf{u}_{21}\right)\left(\mathbf{c}_{r 12} \times \mathbf{c}_{r 11}\right)\right]^{T}
\end{array}\right]
$$

$$
\alpha_{13}^{1}=\left[\begin{array}{cc}
{\left[\left(\left(\mathbf{c}_{r 22} \times \mathbf{c}_{r 23}\right)^{T} \mathbf{c}_{r 12}\right)\left(\mathbf{u}_{11} \times \mathbf{u}_{21}\right)+\left[-\left(\left(\mathbf{u}_{11} \times \mathbf{u}_{21}\right)^{T} \mathbf{u}_{12}\right)\left(\mathbf{c}_{r 22} \times \mathbf{c}_{r 23}\right)-\right.\right.} \\
\left(\left(\mathbf{c}_{r 21} \times \mathbf{c}_{r 23}\right)^{T} \mathbf{c}_{r 22}\right)\left(\mathbf{u}_{11} \times \mathbf{u}_{12}\right)+ & \left(\left(\mathbf{u}_{11} \times \mathbf{u}_{12}\right)^{T} \mathbf{u}_{22}\right)\left(\mathbf{c}_{r 21} \times \mathbf{c}_{r 23}\right)- \\
\left(\left(\mathbf{c}_{r 21} \times \mathbf{c}_{r 12}\right)^{T} \mathbf{c}_{r 23}\right)\left(\mathbf{u}_{11} \times \mathbf{u}_{22}\right)+ & \left(\left(\mathbf{u}_{11} \times \mathbf{u}_{22}\right)^{T} \mathbf{u}_{23}\right)\left(\mathbf{c}_{r 21} \times \mathbf{c}_{r 12}\right)- \\
\left(\left(\mathbf{c}_{r 21} \times \mathbf{c}_{r 22}\right)^{T} \mathbf{c}_{r 12}\right)\left(\mathbf{u}_{11} \times \mathbf{u}_{23}\right)+ & \left(\left(\mathbf{u}_{11} \times \mathbf{u}_{23}\right)^{T} \mathbf{u}_{12}\right)\left(\mathbf{c}_{r 21} \times \mathbf{c}_{r 22}\right)- \\
\left(\left(\mathbf{c}_{r 11} \times \mathbf{c}_{r 23}\right)^{T} \mathbf{c}_{r 22}\right)\left(\mathbf{u}_{21} \times \mathbf{u}_{12}\right)+ & \left(\left(\mathbf{u}_{21} \times \mathbf{u}_{12}\right)^{T} \mathbf{u}_{22}\right)\left(\mathbf{c}_{r 11} \times \mathbf{c}_{r 23}\right)- \\
\left(\left(\mathbf{c}_{r 23} \times \mathbf{c}_{r 12}\right)^{T} \mathbf{c}_{r 11}\right)\left(\mathbf{u}_{21} \times \mathbf{u}_{22}\right)+ & \left(\left(\mathbf{u}_{21} \times \mathbf{u}_{22}\right)^{T} \mathbf{u}_{11}\right)\left(\mathbf{c}_{r 23} \times \mathbf{c}_{r 12}\right)- \\
\left(\left(\mathbf{c}_{r 12} \times \mathbf{c}_{r 22}\right)^{T} \mathbf{c}_{r 11}\right)\left(\mathbf{u}_{21} \times \mathbf{u}_{23}\right)+ & \left(\left(\mathbf{u}_{21} \times \mathbf{u}_{23}\right)^{T} \mathbf{u}_{11}\right)\left(\mathbf{c}_{r 12} \times \mathbf{c}_{r 22}\right)- \\
\left(\left(\mathbf{c}_{r 11} \times \mathbf{c}_{r 21}\right)^{T} \mathbf{c}_{r 23}\right)\left(\mathbf{u}_{12} \times \mathbf{u}_{22}\right)+ & \left(\left(\mathbf{u}_{12} \times \mathbf{u}_{22}\right)^{T} \mathbf{u}_{23}\right)\left(\mathbf{c}_{r 11} \times \mathbf{c}_{r 21}\right)- \\
\left(\left(\mathbf{c}_{r 11} \times \mathbf{c}_{r 22}\right)^{T} \mathbf{c}_{r 21}\right)\left(\mathbf{u}_{12} \times \mathbf{u}_{23}\right)+ & \left(\left(\mathbf{u}_{12} \times \mathbf{u}_{23}\right)^{T} \mathbf{u}_{21}\right)\left(\mathbf{c}_{r 11} \times \mathbf{c}_{r 22}\right)- \\
\left.\left(\left(\mathbf{c}_{r 12} \times \mathbf{c}_{r 11}\right)^{T} \mathbf{c}_{r 21}\right)\left(\mathbf{u}_{22} \times \mathbf{u}_{23}\right)\right]^{T} & \left.\left(\left(\mathbf{u}_{22} \times \mathbf{u}_{23}\right)^{T} \mathbf{u}_{21}\right)\left(\mathbf{c}_{r 12} \times \mathbf{c}_{r 11}\right)\right]^{T}
\end{array}\right]
$$




$$
\alpha_{23}^{1}=\left[\begin{array}{cc}
{\left[\left(\left(\mathbf{c}_{r 22} \times \mathbf{c}_{r 13}\right)^{T} \mathbf{c}_{r 12}\right)\left(\mathbf{u}_{11} \times \mathbf{u}_{21}\right)+\left[-\left(\left(\mathbf{u}_{11} \times \mathbf{u}_{21}\right)^{T} \mathbf{u}_{12}\right)\left(\mathbf{c}_{r 22} \times \mathbf{c}_{r 13}\right)-\right.\right.} \\
\left(\left(\mathbf{c}_{r 21} \times \mathbf{c}_{r 13}\right)^{T} \mathbf{c}_{r 22}\right)\left(\mathbf{u}_{11} \times \mathbf{u}_{12}\right)+ & \left(\left(\mathbf{u}_{11} \times \mathbf{u}_{12}\right)^{T} \mathbf{u}_{22}\right)\left(\mathbf{c}_{r 21} \times \mathbf{c}_{r 13}\right)- \\
\left(\left(\mathbf{c}_{r 21} \times \mathbf{c}_{r 12}\right)^{T} \mathbf{c}_{r 13}\right)\left(\mathbf{u}_{11} \times \mathbf{u}_{22}\right)+ & \left(\left(\mathbf{u}_{11} \times \mathbf{u}_{22}\right)^{T} \mathbf{u}_{13}\right)\left(\mathbf{c}_{r 21} \times \mathbf{c}_{r 12}\right)- \\
\left(\left(\mathbf{c}_{r 21} \times \mathbf{c}_{r 22}\right)^{T} \mathbf{c}_{r 12}\right)\left(\mathbf{u}_{11} \times \mathbf{u}_{13}\right)+ & \left(\left(\mathbf{u}_{11} \times \mathbf{u}_{13}\right)^{T} \mathbf{u}_{12}\right)\left(\mathbf{c}_{r 21} \times \mathbf{c}_{r 22}\right)- \\
\left(\left(\mathbf{c}_{r 11} \times \mathbf{c}_{r 13}\right)^{T} \mathbf{c}_{r 22}\right)\left(\mathbf{u}_{21} \times \mathbf{u}_{12}\right)+ & \left(\left(\mathbf{u}_{21} \times \mathbf{u}_{12}\right)^{T} \mathbf{u}_{22}\right)\left(\mathbf{c}_{r 11} \times \mathbf{c}_{r 13}\right)- \\
\left(\left(\mathbf{c}_{r 13} \times \mathbf{c}_{r 12}\right)^{T} \mathbf{c}_{r 11}\right)\left(\mathbf{u}_{21} \times \mathbf{u}_{22}\right)+ & \left(\left(\mathbf{u}_{21} \times \mathbf{u}_{22}\right)^{T} \mathbf{u}_{11}\right)\left(\mathbf{c}_{r 13} \times \mathbf{c}_{r 12}\right)- \\
\left(\left(\mathbf{c}_{r 12} \times \mathbf{c}_{r 22}\right)^{T} \mathbf{c}_{r 11}\right)\left(\mathbf{u}_{21} \times \mathbf{u}_{13}\right)+ & \left(\left(\mathbf{u}_{21} \times \mathbf{u}_{13}\right)^{T} \mathbf{u}_{11}\right)\left(\mathbf{c}_{r 12} \times \mathbf{c}_{r 22}\right)- \\
\left(\left(\mathbf{c}_{r 11} \times \mathbf{c}_{r 21}\right)^{T} \mathbf{c}_{r 13}\right)\left(\mathbf{u}_{12} \times \mathbf{u}_{22}\right)+ & \left(\left(\mathbf{u}_{12} \times \mathbf{u}_{22}\right)^{T} \mathbf{u}_{13}\right)\left(\mathbf{c}_{r 11} \times \mathbf{c}_{r 21}\right)- \\
\left(\left(\mathbf{c}_{r 11} \times \mathbf{c}_{r 22}\right)^{T} \mathbf{c}_{r 21}\right)\left(\mathbf{u}_{12} \times \mathbf{u}_{13}\right)+ & \left(\left(\mathbf{u}_{12} \times \mathbf{u}_{13}\right)^{T} \mathbf{u}_{21}\right)\left(\mathbf{c}_{r 11} \times \mathbf{c}_{r 22}\right)- \\
\left.\left(\left(\mathbf{c}_{r 12} \times \mathbf{c}_{r 11}\right)^{T} \mathbf{c}_{r 21}\right)\left(\mathbf{u}_{22} \times \mathbf{u}_{13}\right)\right]^{T} & \left.\left(\left(\mathbf{u}_{22} \times \mathbf{u}_{13}\right)^{T} \mathbf{u}_{21}\right)\left(\mathbf{c}_{r 12} \times \mathbf{c}_{r 11}\right)\right]^{T}
\end{array}\right]
$$

Similarly using Gaussian Elimination Algorithm, ${ }^{a} \mathbf{t}^{s}$ is solved and can be expressed similarly to Eq. (54) for any $s=$ $1, \ldots, C_{6}^{8}$ combination.

\section{References}

[1] Kawamura, S., Kino, H., and Won, C., 2000. "High-speed manipulation by using parallel wire-driven robots". Robotica, 18(1), pp. 13-21.

[2] Lambert, C., Nahon, M., and Chalmers, D., 2007. "Implementation of an aerostat positioning system with cable control". IEEE/ASME Transactions on Mechatronic, 12(1), pp. 32-40.

[3] Holland, C. S., and Cannon, D. J., 2004. Cable array robot for material handling, Nov. 30. US Patent 6,826,452.

[4] Alamdari, A., Haghighi, R., and Krovi, V., 2018. "Stiffness modulation in an elastic articulated-cable leg-orthosis emulator: Theory and experiment". IEEE Transactions on Robotics, 34(5), pp. 1266-1279.

[5] Bruckmann, T., Lalo, W., Sturm, C., Schramm, D., and Hiller, M. "Design and realization of a high rack storage and retrieval machine based on wire robot technology". Proceedings of the IDETC/CIE, Portland, Oregan, August 4-7, 2013, pp. 771-780.

[6] Pott, A., Meyer, C., and Verl, A. "Large-scale assembly of solar power plants with parallel cable robots". Robotics (ISR), 2010 41st International Symposium on and 2010 6th German Conference on Robotics (ROBOTIK), Munich, Germany, June 7-9, 2010, pp. 1-6.

[7] Miermeister, P., Lächele, M., Boss, R., Masone, C., Schenk, C., Tesch, J., Kerger, M., Teufel, H., Pott, A., and Bülthoff, H. H. "The cablerobot simulator large scale motion platform based on cable robot technology". IEEE/RSJ International Conference on Intelligent Robots and Systems (IROS), Daejeon, Korea, October 9-14, 2016, pp. 3024-3029.

[8] Merlet, J.-p., and Daney, D. "A portable, modular parallel wire crane for rescue operations". IEEE International Conference on Robotics and Automation (ICRA), Anchorage, Alaska, May 4 - 8, 2010, pp. 2834-2839.

[9] Maeda, K., Tadokoro, S., Takamori, T., Hiller, M., and Verhoeven, R., 1999. "On design of a redundant wire-driven parallel robot warp manipulator". IEEE International Conference on Robotics and Automation, 2, pp. 895-900.

[10] Wischnitzer, Y., Shvalb, N., and Shoham, M., 2008. "Wire-driven parallel robot: Permitting collisions between wires". The International Journal of Robotics Research, 27(9), pp. 1007-1026.

[11] Gagliardini, L., Caro, S., Gouttefarde, M., and Girin, A., 2015. "A reconfiguration strategy for reconfigurable cabledriven parallel robots". IEEE International Conference on Robotics and Automation (ICRA), Washington, USA, May 26-30, 2015, pp. 1613-1620.

[12] Gagliardini, L., Caro, S., Gouttefarde, M., and Girin, A., 2016. "Discrete reconfiguration planning for cable-driven parallel robots". Mechanism and Machine Theory, 100, pp. 313-337.

[13] Nguyen, D. Q., Gouttefarde, M., Company, O., and Pierrot, F. "On the analysis of large-dimension reconfigurable suspended cable-driven parallel robots". IEEE International Conference on Robotics and Automation (ICRA), Hong Kong, May 31 to June 5, 2014, pp. 5728-5735.

[14] Zhou, X., Tang, C. P., and Krovi, V. "Analysis framework for cooperating mobile cable robots". 2012 IEEE International Conference on Robotics and Automation (ICRA), Minnesota USA, May 14-18, 2012, pp. 3128-3133.

[15] Anson, M., Alamdari, A., and Krovi, V., 2017. "Orientation workspace and stiffness optimization of cable-driven parallel manipulators with base mobility". ASME J Mech Robot, 9(3), p. 031011.

[16] Sovizi, J., Rai, R., and Krovi, V., 2018. "Wrench uncertainty quantification and reconfiguration analysis in loosely interconnected cooperative systems". ASCE-ASME Journal of Risk and Uncertainty in Engineering Systems, Part B: Mechanical Engineering, 4(2), p. 021002.

[17] Rasheed, T., Long, P., Marquez-Gamez, D., and Caro, S. "Tension distribution algorithm for planar mobile cable-driven parallel robots". The Third International Conference on Cable-Driven Parallel Robots (CableCon 2017), Quebec City, Canada, August 2-4, 2017, pp. 268-279.

[18] Pedemonte, N., Rasheed, T., Marquez-Gamez, D., Long, P., Hocquard, É., Babin, F., Fouché, C., Caverot, G., Girin, 
A., and Caro, S., 2020. "Fastkit: A mobile cable-driven parallel robot for logistics". In Advances in Robotics Research: From Lab to Market. Springer, pp. 141-163.

[19] Gouttefarde, M., Daney, D., and Merlet, J.-P., 2011. "Interval-analysis-based determination of the wrench-feasible workspace of parallel cable-driven robots". IEEE Transactions on Robotics, 27(1), pp. 1-13.

[20] Bosscher, P., Riechel, A. T., and Ebert-Uphoff, I., 2006. "Wrench-feasible workspace generation for cable-driven robots". IEEE Transactions on Robotics, 22(5), pp. 890-902.

[21] BROWN, G., 1985. "Skycam: An aerial robotic camera system". BYTE, p. 122.

[22] Merlet, J.-P. "Kinematics of the wire-driven parallel robot marionet using linear actuators". IEEE International Conference on Robotics and Automation (ICRA), Pasadena, California, May 19-23, 2008, pp. 3857-3862.

[23] Kawamura, S., 1995. "Development of an ultrahigh speed robot falcon using wire drive system". Robotics and Automation, pp. 215-220.

[24] Albus, J., Bostelman, R., and Dagalakis, N., 1993. "The nist robocrane”. Journal of Field Robotics, 10(5), pp. 709-724.

[25] Bouchard, S., Gosselin, C., and Moore, B., 2010. "On the ability of a cable-driven robot to generate a prescribed set of wrenches". ASME J Mech Robot, 2(1), p. 011010.

[26] Grünbaum, B., 2003. "Convex polytopes. 2003”. Grad. Texts in Math.

[27] Kawamura, S., and Ito, K. "A new type of master robot for teleoperation using a radial wire drive system". In IEEE/RSJ International Conference on Intelligent Robots and Systems (IROS). Yokohama, Japan, July 26-30, 1993, pp. 55-60.

[28] Hiller, M., Fang, S., Mielczarek, S., Verhoeven, R., and Franitza, D., 2005. "Design, analysis and realization of tendon-based parallel manipulators". Mechanism and Machine Theory, 40(4), pp. 429-445.

[29] Lafaye, J., Gouaillier, D., and Wieber, P.-B. "Linear model predictive control of the locomotion of pepper, a humanoid robot with omnidirectional wheels". IEEE-RAS International Conference on Humanoid Robots, Madrid, Spain, November 18-20, 2014, pp. 336-341.

[30] Sardain, P., and Bessonnet, G., 2004. "Forces acting on a biped robot center of pressure-zero moment point". IEEE Transactions on Systems, Man, and Cybernetics-Part A: Systems and Humans, 34(5), pp. 630-637.

[31] Vukobratović, M., and Borovac, B., 2004. "Zero-moment point-thirty five years of its life". International Journal of Humanoid Robotics, 1(01), pp. 157-173.

[32] Rudy, J., 2014. “Zero-moment point walking controller for humanoid walking using darwin-op". Dept. of Aerospace and Mechanical Engineering, University of Notre Dame, Indiana.

[33] Samy, V., Caron, S., Bouyarmane, K., and Kheddar, A., 2017. "Adaptive compliance in post-impact humanoid falls using preview control of a reduce model".

[34] Caron, S., Pham, Q.-C., and Nakamura, Y., 2017. "Zmp support areas for multicontact mobility under frictional constraints". IEEE Transactions on Robotics, 33(1), pp. 67-80.

[35] Rasheed, T., Long, P., Marquez-Gamez, D., and Caro, S. "Available wrench set for planar mobile cable-driven parallel robots". IEEE International Conference on Robotics and Automation (ICRA), Brisbane, Australia, May 20-25, 2018, pp. $962-967$.

[36] Motzkin, T. S., 1953. "The double description method, in contributions to the theory of games ii". Annals of Mathematics Study, 28.

[37] Barber, C. B., Dobkin, D. P., and Huhdanpaa, H., 1996. "The quickhull algorithm for convex hulls". ACM Transactions on Mathematical Software (TOMS), 22(4), pp. 469-483.

[38] Poole, D., 2014. Linear algebra: A modern introduction. Cengage Learning.

[39] Bosscher, P., Riechel, A. T., and Ebert-Uphoff, I., 2006. "Wrench-feasible workspace generation for cable-driven robots". IEEE Transactions on Robotics, 22(5), pp. 890-902.

[40] Guay, F., Cardou, P., Cruz-Ruiz, A. L., and Caro, S., 2013. "Measuring how well a structure supports varying external wrenches". New Advances in Mechanisms, Transmissions and Applications, Basque Country, Bilbao, Spain, 2013, pp. 385-392.

[41] Ruiz, A. L. C., Caro, S., Cardou, P., and Guay, F. "Arachnis: Analysis of robots actuated by cables with handy and neat interface software". In Pott A., Bruckmann T. (eds) Cable-Driven Parallel Robots Mechanisms and Machine Science, Vol. 32. Springer, Cham, pp. 293-305. 


\section{List of Figures}

1 FASTKIT prototype (a) Navigation mode (b) Undeployed configuration (c) Deployed configuration at the

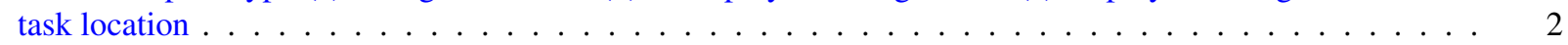

2 Methodology used to determine the WFW of a MCDPR . . . . . . . . . . . . . . . . 3

3 (a) A MCDPR with eight cables $(m=8)$ and four mobile bases $(p=4)$. Its moving-platform has six degreeof-freedom $(\mathrm{n}=6)(\mathrm{b}) j$ th mobile base with four wheels $\left(c_{j}=4\right) \ldots \ldots \ldots$

$4 \quad$ (a) Footprint of $\mathscr{M}_{j}$ with $c_{j}=6$ wheels (b) Linearized friction pyramid at ZMP $\left(C_{j}\right) . \ldots . . . . .$.

5 Tension space associated to (a) $\mathscr{M}_{1}$, (b) $\mathscr{M}_{2}$,(c) $\mathscr{M}_{3}$, (d) $\mathscr{M}_{4}$ considering both the cable tension limits and the static equilibrium of the mobile bases . . . . . . . . . . . . . . . . . . . .

6 (a) Planar MCDPR with one point-mass end-effector, two mobile bases and four cables (b) Static workspace (c,d) Modified cable tension space (e) $\mathscr{V}$-Representation of AWS of a CDPR (in black) and MCDPR (in green) (f) $\mathscr{H}-$ Representation of the AWS formed by the intersection of the hyperplanes . . . . . . . . . .

7 (a) Configuration under study of $p=2, m=4$ and $n=3$ MCDPR (b) Comparison of AWS between CDPR (in black + green) and MCDPR (in green) . . . . . . . . . . . . . . . . . . . . . . . . 16

$8 \quad$ (a) $\mathscr{T}_{1}$ and (b) $\mathscr{T}_{2}$ for MCDPR configuration in Fig. $7($ a) $\ldots \ldots \ldots$

9 Correspondence between the WFW facets obtained with the Convex Hull approach (in green) and those obtained with HSM: (a,b) Mobile Base tipping; (c,d) Mobile Base sliding for the moving-platform pose shown in Fig. 7(a) . . . . . . . . . . . . . . . . . . . . . . . . . . . .

10 WFW of FASTKIT at different configuration of mobile bases with a constant moving-platform orientation . 